\title{
Implementing Modified Burg Algorithms in Multivariate Subset Autoregressive Modeling
}

\author{
A. Alexandre Trindade*
}

February 3, 2003

\begin{abstract}
The large number of parameters in subset vector autoregressive models often leads one to procure fast, simple, and efficient alternatives or precursors to maximum likelihood estimation. We present the solution of the multivariate subset Yule-Walker equations as one such alternative. In recent work, Brockwell, Dahlhaus, and Trindade (2002), show that the Yule-Walker estimators can actually be obtained as a special case of a general recursive Burg-type algorithm. We illustrate the structure of this Algorithm, and discuss its implementation in a high-level programming language. Applications of the Algorithm in univariate and bivariate modeling are showcased in examples. Univariate and bivariate versions of the Algorithm written in Fortran 90 are included in the appendix, and their use illustrated.
\end{abstract}

Keywords: binary tree, Fortran 90, pointer linked list, recursive algorithm, Yule-Walker estimation

\section{Introduction}

AutoRegressive Moving Average (ARMA) models are well-known and popular in the time series literature. Among others, they are extensively used to model economic and electrical systems whose evolution in time (modulo preliminary transformations, de-trending, and de-seasonalizing) can be well approximated by that of a stationary process. Stationarity constrains a process to have second order properties that do not evolve with time, i.e.

${ }^{*}$ Department of Statistics, University of Florida, P.O. Box 118545, Gainesville, FL 32611-8545 (trindade@stat.ufl.edu) 
constant mean, and a covariance structure between any two observations that depends only on the distance in time (the lag) separating them.

In the multivariate setting, one attempts to model the joint behavior of several univariate series over the same span of time. The usefulness of multivariate ARMA models here though, is stymied by identifiability issues concerning the model parameters (see for example Brockwell and Davis 1991, sec. 11.5). A common alternative is to restrict attention to two special cases: invertible Moving Average (MA) models, and causal AutoRegressive (AR) models, whose parameters are uniquely determined by the second order properties of the process. AR models (called Vector Autoregressive (VAR) in the multivariate case) are often favored over MA models due to their interpretability, simplicity of estimation, and ease of forecasting. They are extensively used in signal processing for modeling various phenomena associated with speech and audio; see for example Godsill and Rayner (1998).

The $d$-dimensional vector process $\left\{\mathbf{X}_{t}, t=0, \pm 1, \ldots\right\}$, is said to be a VAR process of order $p, \operatorname{VAR}(p)$, if it is a stationary solution of the equations,

$$
\mathbf{X}_{t}=\Phi(1) \mathbf{X}_{t-1}+\cdots+\Phi(p) \mathbf{X}_{t-p}+\mathbf{Z}_{t}
$$

where, $\Phi(1), \ldots, \Phi(p)$, are $(d \times d)$ constant matrices (the VAR coefficient matrices), and $\left\{\mathbf{Z}_{t}\right\}$ is a sequence of zero-mean uncorrelated random vectors, each with covariance matrix $\Sigma$. We call the process $\left\{\mathbf{Z}_{t}\right\}$ white noise, and write $\left\{\mathbf{Z}_{t}\right\} \sim \mathrm{WN}(\mathbf{0}, \Sigma)$. The autocovariance function of $\mathbf{X}_{t}$ is,

$$
E\left[\mathbf{X}_{t+h} \mathbf{X}_{t}^{\prime}\right]=\Gamma(h), \quad h=0, \pm 1, \ldots
$$

$\mathrm{A} \operatorname{VAR}(p)$ is therefore a linear regression of the current value of the series on its previous $p$ values i.e. an autoregression. We say that we are modeling the series on the lagged set $\{1, \ldots, p\}$.

One can generalize this to modeling on a lagged subset

$$
K=\left\{k_{1}, \ldots, k_{m}\right\} \subseteq\{1, \ldots, p\}, \quad \text { with } k_{1}<\cdots<k_{m} \equiv p,
$$

and the coefficient matrices pertaining to the lags not present in the set $K$, constrained to be zero. Such models are called Subset Vector AutoRegressive (SVAR; SAR in the univariate case), and take the form

$$
\mathbf{X}_{t}=\Phi_{K}\left(k_{1}\right) \mathbf{X}_{t-k_{1}}+\cdots+\Phi_{K}\left(k_{m}\right) \mathbf{X}_{t-k_{m}}+\mathbf{Z}_{t}, \quad\left\{\mathbf{Z}_{t}\right\} \sim \mathrm{WN}\left(\mathbf{0}, U_{K}\right) .
$$

SVAR models are appropriate in situations where one does not wish to include all the lags of the complete (full-set) VAR model. Two such instances are: 
Modeling of seasonal time series. If $B$ denotes the backward shift operator, i.e. $B^{k} X_{t}=X_{t-k}$ for any positive integer $k$, then causal SAR models of the form,

$$
\left(1-\psi B^{s}\right)\left(1-\phi_{1} B-\cdots-\phi_{p} B^{p}\right) X_{t}=Z_{t},
$$

will exhibit approximate cyclical behavior for appropriate values of the coefficients $\psi, \phi_{1}, \ldots, \phi_{p}$, and orders $s, p$, as evidenced by sharp peaks in the spectral density. This suggests that some seasonal time series can effectively be modeled as SAR processes.

Figure 1 shows a realization from the $\operatorname{SAR}(3)$,

$$
X_{t}-0.99 X_{t-3}=Z_{t},\left\{Z_{t}\right\} \sim \mathrm{WN}(0,1),
$$

along with a plot of the spectral density function of the process on the interval $(0, \pi)$. The spectral density peaks at a frequency of $2 \pi / 3$ radians per unit time, which corresponds to a period of length 3 .

Fitting best subset models. As in linear regression, one can search for the "best" subset AR/VAR model up to some maximum order, $p$. "Best" can be measured by one's favorite information criterion, such as Akaike (AIC), Bayesian (BIC), Schwarz (SIC), or even Minimum Description Length (MDL). Researchers have devised efficient algorithms to perform this search. One of the earliest attempts was made by McClave (1975), who used an algorithm adapted from linear regression. Penm and Terrell (1982), introduced an algorithm recursive in the maximum lag for best subset identification. Zhang and Terrell (1997) refine the search by inspecting certain statistics. Rather than performing an exhaustive search through all $2^{p}$ models, Sarkar and Sharma (1997) propose a statistical method for identifying the best subset.

Figure 2 shows the celebrated Canadian Lynx Trappings data. Ecological oscillations in predator-prey populations, mean that the logarithms of this data set are often modeled as a SAR process; a perennial favorite in the SAR literature. The lower part of the figure shows a spectral density estimator (the periodogram) for this data, which suggests the period of the oscillations to be approximately $2 \pi / 0.6 \approx 10.5$ years. In section 4 , we will apply the algorithm of section 2 to perform an exhaustive search for the best SAR model.

For a given SVAR model order, one typically wishes to find maximum likelihood (ML) estimates of the parameters. Using standard arguments, 
Figure 1: The process $X_{t}-0.99 X_{t-3}=Z_{t},\left\{Z_{t}\right\} \sim \mathrm{WN}(0,1)$. Top: a realization of the process with Gaussian noise. Bottom: the corresponding spectral density function.

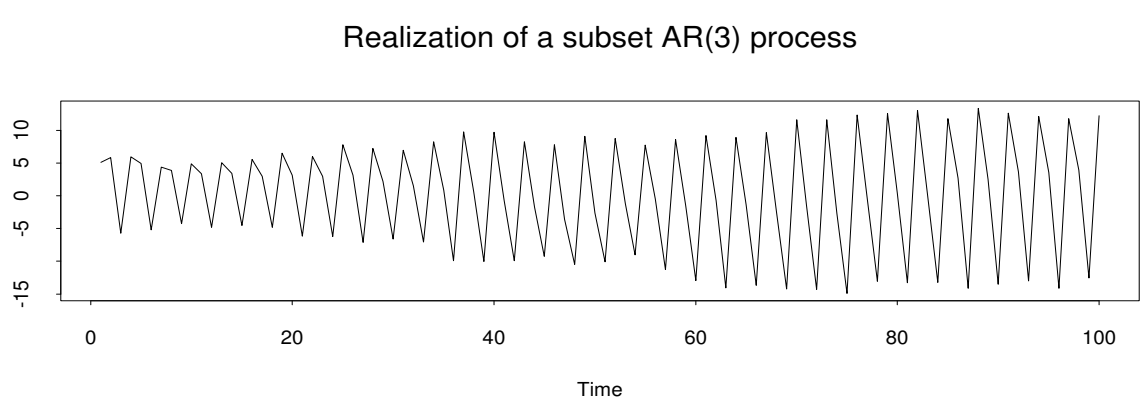

Spectral density function

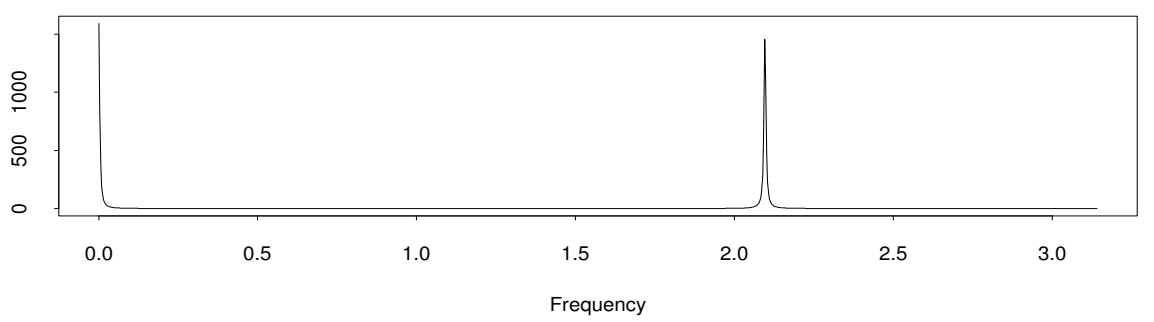

the $-2 \log$ likelihood for the vectors $\mathbf{X}_{1}, \ldots, \mathbf{X}_{n}$ from the Gaussian SVAR process of dimension $d$ defined by equation (1), can be shown to be

$$
\begin{aligned}
& \mathcal{L}\left(\Phi_{K}\left(k_{1}\right), \ldots, \Phi_{K}\left(k_{m}\right), U_{K}\right)=n d \log (2 \pi)+\log \operatorname{det}\left(\Gamma_{k_{m}}\right) \\
& \quad+\left(n-k_{m}\right) \log \operatorname{det}\left(U_{K}\right)+\left[\mathbf{X}_{1}^{\prime}, \ldots, \mathbf{X}_{k_{m}}^{\prime}\right] \Gamma_{k_{m}}^{-1}\left[\mathbf{X}_{1}^{\prime}, \ldots, \mathbf{X}_{k_{m}}^{\prime}\right]^{\prime} \\
& \quad+\sum_{t=k_{m}+1}^{n}\left[\mathbf{X}_{t}-\sum_{j \in K} \Phi_{K}(j) \mathbf{X}_{t-j}\right]^{\prime} U_{K}^{-1}\left[\mathbf{X}_{t}-\sum_{j \in K} \Phi_{K}(j) \mathbf{X}_{t-j}\right]
\end{aligned}
$$

where $\Gamma_{k_{m}}=\mathbf{E}\left(\left[\mathbf{X}_{1}^{\prime}, \ldots, \mathbf{X}_{k_{m}}^{\prime}\right]^{\prime}\left[\mathbf{X}_{1}^{\prime}, \ldots, \mathbf{X}_{k_{m}}^{\prime}\right]\right)$.

\section{Remark 1}

The potentially large number of parameters involved in $M L$ estimation 
Figure 2: The annual Canadian lynx trappings data. Top: numbers of lynx trapped between 1821 and 1934. Bottom: the periodogram of the data.

Canadian lynx trappings data

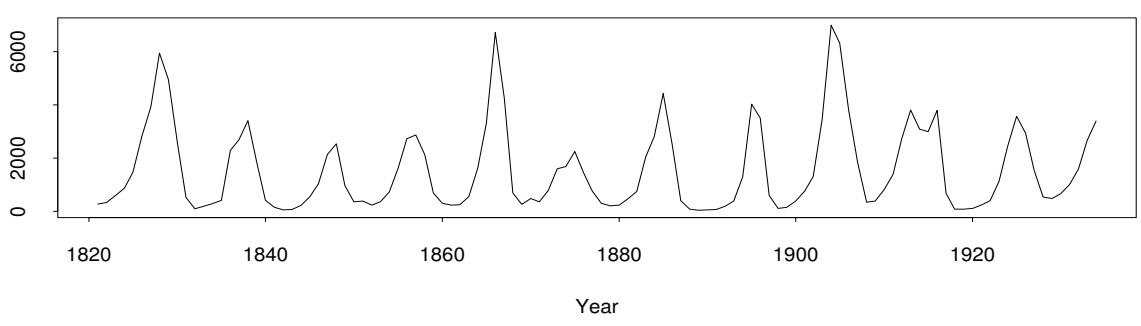

Periodogram

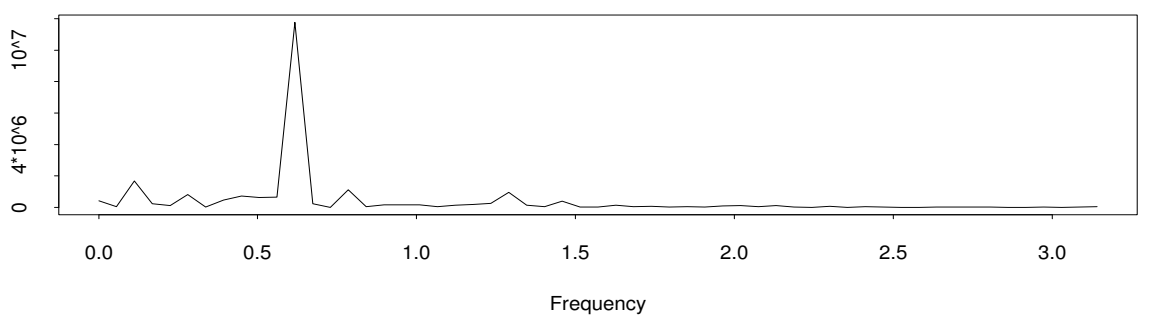

$\left(d^{2} k_{m}+\frac{d^{2}+d}{2}\right.$ of them), and the possible existence of many local minima which are much larger than the global minimum, makes the numerical search for the minimizers a difficult problem. The feasibility of ML estimation is therefore highly dependent upon good initial estimates.

For this, and the reason that one may wish to avoid ML estimation altogether, it is important to consider alternative fast and simple SVAR estimation methods for obtaining models with high likelihoods. Recently, Brockwell, Dahlhaus, and Trindade (2002), introduced a method for doing just that. Their method, the BDT Algorithm which we consider in section 2 , is recursive in the model order, parameter estimates of larger order models being constructed from those of smaller order models. Since the Brockwell et al. (2002) paper focuses mostly on theoretical aspects, the main purpose of this article is to serve as a pragmatic complement to it in the following 
ways:

(i) Elucidate the recursive structure of the Algorithm.

(ii) Discuss the main issues involved in implementing the Algorithm in a high-level programming language like Fortran 90.

(iii) Provide coded versions of the Algorithm along with examples that illustrate its usage.

Section 3 accomplishes the first two goals, in the framework of a binary tree of pointer-linked nodes. The examples are presented in Section 4, which also illustrates some meta applications of the Algorithm. (Accompanying data sets are provided in Appendix B.) Fortran 90 programs implementing a univariate (BDT.F90) and a bivariate (BDT2.F90) version of the Algorithm are provided in Appendix C. Appendix A summarizes the function of the principal subroutines in these programs.

\section{Estimation Methods}

In this section we discuss alternative SVAR parameter estimation methods to ML. The first is a generalization of the well-known Yule-Walker method of moments estimator for full-set modeling, and has not previously appeared in the literature in this form. The second is a flexible recursive Burg-type algorithm, introduced by Brockwell et al. (2002), whose structure and implementation is the main focus of this paper. In order to introduce both methods, we will need to consider not only the (forward) SVAR model (1), but also the backward SVAR model

$$
\mathbf{X}_{t}=\sum_{j \in K^{*}} \Psi_{K^{*}}(j) \mathbf{X}_{t+j}+\mathbf{Z}_{t}, \quad\left\{\mathbf{Z}_{t}\right\} \sim \mathrm{WN}\left(\mathbf{0}, V_{K^{*}}\right)
$$

where $K^{*}=\left\{k_{m}-k_{m-1}, \ldots, k_{m}-k_{1}, k_{m}\right\}$, and suppose that the process $\left\{\mathbf{X}_{t}\right\}$ is causal (meaning that the current value of the series can be expressed as a function of current and past values of the white noise sequence as, $\left.\mathbf{X}_{t}=\sum_{j=0}^{\infty} \Upsilon_{j} \mathbf{Z}_{t-j}\right)$.

\subsection{Non-Recursive Estimation: The Yule-Walker Equations}

If we multiply both sides of (1) by $\mathbf{X}_{t-i}, i=0, k_{1}, \ldots, k_{m}$ in turn, and (noting the causal representation) take expectations, we obtain the so-called 
Yule-Walker (YW) equations:

$$
\begin{aligned}
\Gamma(k) & =\sum_{j \in K} \Phi_{K}(j) \Gamma(k-j), \quad k \in K, \\
U_{K} & =\Gamma(0)-\sum_{j \in K} \Phi_{K}(j) \Gamma(j)^{\prime} .
\end{aligned}
$$

For the backward SVAR model (3), the corresponding YW equations are

$$
\begin{aligned}
\Gamma(k)^{\prime} & =\sum_{j \in K^{*}} \Psi_{K^{*}}(j) \Gamma(j-k), \quad k \in K^{*}, \\
V_{K^{*}} & =\Gamma(0)-\sum_{j \in K^{*}} \Psi_{K^{*}}(j) \Gamma(j) .
\end{aligned}
$$

Now define $R_{K}$ and $G_{K}$ to be matrices of autocovariances as follows: with $k_{0}=0$, define the $(i, j)$ th $, i, j=1, \ldots, m+1$, block-matrix entry of $R_{K}$ to be,

$$
\left(R_{K}\right)_{(i, j)}=\left\{\begin{array}{ll}
\Gamma\left(k_{j-1}-k_{i-1}\right), & j \geq i \\
\Gamma\left(k_{j-1}-k_{i-1}\right)^{\prime}, & j<i
\end{array},\right.
$$

and $G_{K}$ obtained from $R_{K}$ by striking out the first block row and column. The $m+1$ forward YW equations can now be succinctly written in blockmatrix form as,

$$
\left[I_{d},-\Phi_{K}\left(k_{1}\right),-\Phi_{K}\left(k_{2}\right), \ldots,-\Phi_{K}\left(k_{m-1}\right),-\Phi_{K}\left(k_{m}\right)\right] R_{K}=\left[U_{K}, 0, \ldots, 0\right],
$$

and the backward YW equations as,

$\left[-\Psi_{K^{*}}\left(k_{m}\right),-\Psi_{K^{*}}\left(k_{m}-k_{1}\right), \ldots,-\Psi_{K^{*}}\left(k_{m}-k_{m-1}\right), I_{d}\right] R_{K}=\left[0, \ldots, 0, V_{K^{*}}\right]$.

Defining

$$
\Gamma_{K} \equiv\left[\Gamma\left(k_{1}\right), \Gamma\left(k_{2}\right), \ldots, \Gamma\left(k_{m-1}\right), \Gamma\left(k_{m}\right)\right]
$$

and

$$
\Phi_{K} \equiv\left[\Phi_{K}\left(k_{1}\right), \Phi_{K}\left(k_{2}\right), \ldots, \Phi_{K}\left(k_{m-1}\right), \Phi_{K}\left(k_{m}\right)\right],
$$

we can write (4)-(5) in the reduced block-matrix form

$$
\begin{aligned}
\Gamma_{K} & =\Phi_{K} G_{K}, \\
U_{K} & =\Gamma(0)-\Phi_{K} \Gamma_{K}^{\prime} .
\end{aligned}
$$

These can now be solved for $\Phi_{K}$ and $U_{K}$ :

$$
\begin{aligned}
\Phi_{K} & =\Gamma_{K} G_{K}^{-1}, \\
U_{K} & =\Gamma(0)-\Gamma_{K} G_{K}^{-1} \Gamma_{K}^{\prime},
\end{aligned}
$$


where $G_{K}^{-1}$ denotes any generalized inverse of $G_{K}$. The solution $\Phi_{K}$, gives the minimum mean-squared error linear predictor of $\mathbf{X}_{t}$ in terms of $\mathbf{X}_{t-i}, i \in$ $K$. Its mean-squared error is $U_{K}$. Analogous results hold for the backward YW equations.

When fitting SVAR model (1) to a set of observations $\mathbf{x}_{1}, \ldots, \mathbf{x}_{n}$ from the zero-mean random vectors $\mathbf{X}_{1}, \ldots, \mathbf{X}_{n}$, one of the simplest approaches is to substitute sample estimates for the autocovariances in (10) and (11). Taking the usual estimator of the autocovariance matrix at lag $h$ to be,

$$
\hat{\Gamma}(h)= \begin{cases}\frac{1}{n} \sum_{t=1}^{n-h} \mathbf{x}_{t+h} \mathbf{x}_{t}^{\prime} & , \text { if } h \geq 0, \\ \hat{\Gamma}(-h)^{\prime} & , \text { if } h<0\end{cases}
$$

the resulting method of moments estimates are,

$$
\begin{aligned}
\hat{\Phi}_{K} & =\hat{\Gamma}_{K} \hat{G}_{K}^{-1}, \\
\hat{U}_{K} & =\hat{\Gamma}(0)-\hat{\Phi}_{K} \hat{\Gamma}_{K}^{\prime} .
\end{aligned}
$$

These are the so-called YW estimates in the full-set case, and we will refer to their subset generalization by the same name. The fitted YW SVAR model is therefore,

$$
\mathbf{X}_{t}=\hat{\Phi}_{K}\left(k_{1}\right) \mathbf{X}_{t-k_{1}}+\cdots+\hat{\Phi}_{K}\left(k_{m}\right) \mathbf{X}_{t-k_{m}}+\mathbf{Z}_{t}, \quad\left\{\mathbf{Z}_{t}\right\} \sim \mathrm{WN}\left(\mathbf{0}, \hat{U}_{K}\right) .
$$

\subsection{Recursive Estimation: The BDT Algorithm}

By defining the empirical forward and backward prediction error residuals $\hat{\boldsymbol{\varepsilon}}_{K}$ and $\hat{\boldsymbol{\eta}}_{K^{*}}$, associated with models (1) and (3) as,

$$
\hat{\varepsilon}_{K}(t)=\mathbf{x}_{t}-\sum_{i \in K} \hat{\Phi}_{K}(i) \mathbf{x}_{t-i}, \quad \text { and }, \quad \hat{\boldsymbol{\eta}}_{K^{*}}(t)=\mathbf{x}_{t}-\sum_{j \in K^{*}} \hat{\Psi}_{K^{*}}(j) \mathbf{x}_{t+j},
$$

Brockwell et al. (2002), introduce a family of SVAR model parameter estimators, based on Burg's (1968) recursive algorithm. Their BDT Algorithm, takes the following form. 


\section{Algorithm 1 (The BDT Algorithm)}

$$
\begin{aligned}
\hat{\Phi}_{K}\left(k_{m}\right) & =\cdots \\
\hat{\Phi}_{K}(i) & =\hat{\Phi}_{J}(i)-\hat{\Phi}_{K}\left(k_{m}\right) \hat{\Psi}_{J^{*}}\left(k_{m}-i\right), \quad i \in J \\
\hat{\Psi}_{K^{*}}\left(k_{m}\right) & =\hat{V}_{J^{*}} \hat{\Phi}_{K}\left(k_{m}\right)^{\prime} \hat{U}_{J}^{-1} \\
\hat{\Psi}_{K^{*}}(j) & =\hat{\Psi}_{J^{*}}(j)-\hat{\Psi}_{K^{*}}\left(k_{m}\right) \hat{\Phi}_{J}\left(k_{m}-j\right), \quad j \in J^{*} \\
\hat{U}_{K} & =\hat{U}_{J}-\hat{\Phi}_{K}\left(k_{m}\right) \hat{V}_{J^{*}} \hat{\Phi}_{K}\left(k_{m}\right)^{\prime} \\
\hat{V}_{K^{*}} & =\hat{V}_{J^{*}}-\hat{\Psi}_{K^{*}}\left(k_{m}\right) \hat{U}_{J} \hat{\Psi}_{K^{*}}\left(k_{m}\right)^{\prime} \\
\hat{\varepsilon}_{K}(t) & =\hat{\boldsymbol{\varepsilon}}_{J}(t)-\hat{\Phi}_{K}\left(k_{m}\right) \hat{\boldsymbol{\eta}}_{J^{*}}\left(t-k_{m}\right) \\
\hat{\boldsymbol{\eta}}_{K^{*}}(t) & =\hat{\boldsymbol{\eta}}_{J^{*}}(t)-\hat{\Psi}_{K^{*}}\left(k_{m}\right) \hat{\varepsilon}_{J}\left(t+k_{m}\right)
\end{aligned}
$$

with initial conditions,

$$
\begin{aligned}
\hat{\varepsilon}_{\emptyset}(t) & =\hat{\boldsymbol{\eta}}_{\emptyset}(t)= \begin{cases}\mathbf{x}_{t}, & t \in\{1, \ldots, n\} \\
\mathbf{0}, & \text { otherwise }\end{cases} \\
\hat{U}_{\emptyset} & =\hat{\Gamma}(0)=\hat{V}_{\emptyset}
\end{aligned}
$$

and the sets $J$ and $J^{*}$, formed from the sets $K$ and $K^{*}$, respectively, by omitting $k_{m}$.

A variety of different estimators can be obtained by an appropriate selection of the boxed reflection coefficient expression in (16). Brockwell et al. (2002), note that the choice

$$
\hat{\Phi}_{K}\left(k_{m}\right)=\left(\frac{1}{n} \sum_{t=1}^{n+k_{m}} \hat{\varepsilon}_{J}(t) \hat{\boldsymbol{\eta}}_{J^{*}}\left(t-k_{m}\right)^{\prime}\right) \hat{V}_{J^{*}}^{-1},
$$

gives precisely the YW estimators (13) and (14) (reformulated via similar recursions, the resulting Algorithm is known as Levinson-Durbin), but that selecting $\hat{\Phi}_{K}\left(k_{m}\right)$ to be the minimizer of the weighted sum of forward and backward prediction errors

$$
\sum_{t=k_{m}+1}^{n}\left[\hat{\boldsymbol{\varepsilon}}_{K}(t)^{\prime} A \hat{\boldsymbol{\varepsilon}}_{K}(t)+\hat{\boldsymbol{\eta}}_{K^{*}}\left(t-k_{m}\right)^{\prime} B \hat{\boldsymbol{\eta}}_{K^{*}}\left(t-k_{m}\right)\right],
$$

tends to produce models with consistently higher Gaussian likelihoods. By selecting different weight matrices $\mathrm{A}$ and $\mathrm{B}$, they propose a total of three additional methods: Burg, Vieira-Morf, and Nuttall-Strand, each being a 
plausible subset generalization of existing full-set analogues with the same name.

The BDT Algorithm necessarily couples together the forward and backward modeling problems. Arranging the elements of $K$ on the number line as shown in Figure 3, allows us to better visualize this coupling. The for-

Figure 3: The set of lags $K=\left\{k_{1}, \ldots, k_{m}\right\}$ arranged on the number line.

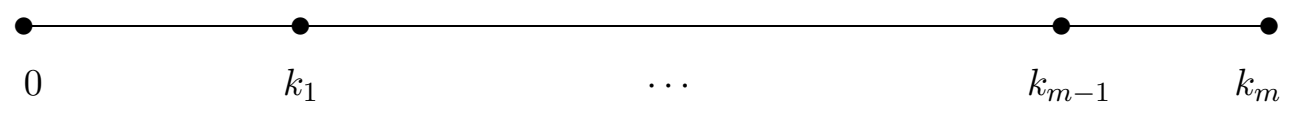

ward set of lags, $K$, are simply the distances of the elements of $K$ from the origin; while the backward set of lags, $K^{*}$, are the corresponding distances from $k_{m}$.

Note that the YW estimator, $\hat{\Phi}_{K}\left(k_{m}\right)$, obtained from (13), requires the inversion of $\hat{G}_{K}$, which is of dimension $m d$. Recursive algorithms are better suited to searching for a best subset model with a specified maximum number of lags, and involve inversion of matrices whose dimension is at most $d$ ( $d^{2}$ in some instances).

\section{Implementing the BDT Algorithm}

Apart from the special case of YW, estimators arising from the BDT Algorithm cannot in general be reformulated in a non-recursive manner. The intricate structure of the recursions, a by-product of the forward and backward model coupling, can seem rather daunting from a programming perspective. In this section therefore, we discuss the main issues involved in implementing this Algorithm in a high-level programming language like Fortran 90. An important goal is to minimize computing time, and our approach will be to create a linked list of nodes in the form of a binary tree. In the process, we will make use of recursive pointers, recursive subroutines, and data types that incorporate recursive definitions.

\subsection{Building a binary tree of linked nodes}

The recursive solution of the equations defining the BDT Algorithm, generates a collection of estimators of SVAR models of increasing orders, until the required order is reached. Suppose modeling on the set of lags $\{1,3,7\}$ 
is desired. To determine where application of the algorithm should begin, we first need to work down to derived subsets of lags comprised of just one lag. This is done by successively forming the $J$ and $J^{*}$ subsets of lags for each parent set of lags $K$, as shown in Figure 4. Each of the subsets $J$ and $J^{*}$ then assumes the role of a parent lag, $K$, and the procedure is repeated. In the resulting binary tree structure, we will refer to all the modeling information pertaining to a set of lags as a node. The number of lags in a node will define its level in the tree. The strategy for this recursive tree-building will then be as follows:

\section{Pseudocode 1 (Build Tree)}

level $:=m$

while level $>1$ do

for each node in current level do

compute lags in $J$ and $J^{*}$ subnodes

direct pointers to $J$ and $J^{*}$ subnodes od

level $:=$ level -1 od

Figure 4: Binary tree of linked nodes for modeling on the set of lags $\{1,3,7\}$.

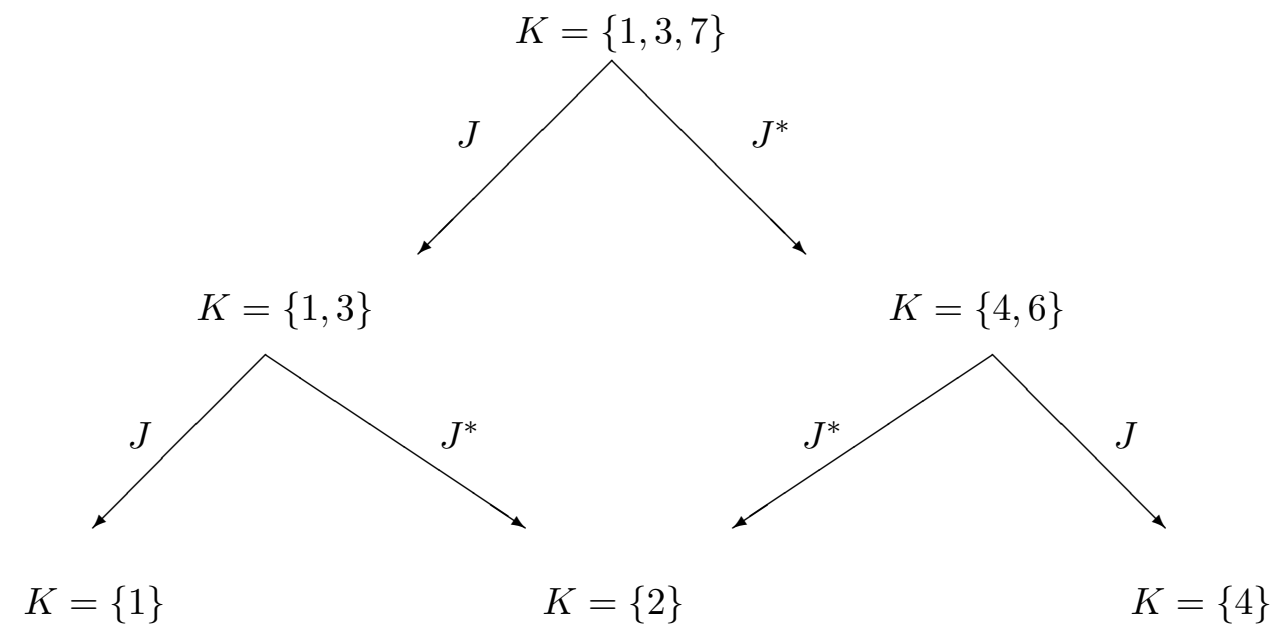


For programming, it will be necessary to rewrite the backward model equations (17), (18), (19), and (21), in the unstarred lags format:

$$
\begin{aligned}
\hat{\Psi}_{K}\left(k_{m}\right) & =\hat{V}_{J} \hat{\Phi}_{K^{*}}\left(k_{m}\right)^{\prime} \hat{U}_{J^{*}}^{-1}, \\
\hat{\Psi}_{K}(j) & =\hat{\Psi}_{J}(j)-\hat{\Psi}_{K}\left(k_{m}\right) \hat{\Phi}_{J^{*}}\left(k_{m}-j\right), \quad j \in J, \\
\hat{V}_{K} & =\hat{V}_{J}-\hat{\Psi}_{K}\left(k_{m}\right) \hat{U}_{J^{*}} \hat{\Psi}_{K}\left(k_{m}\right)^{\prime}, \\
\hat{\boldsymbol{\eta}}_{K}(t) & =\hat{\boldsymbol{\eta}}_{J}(t)-\hat{\Psi}_{K}\left(k_{m}\right) \hat{\varepsilon}_{J^{*}}\left(t+k_{m}\right),
\end{aligned}
$$

obtained by noting that $\left(K^{*}\right)^{*}=K$, and $\left(J^{*}\right)^{*}=J$. The unstarred lags representation carifies how the backward model estimates should be computed for any given node. Letting node be a user-defined derived data type, and $K=\left\{k_{1}, \ldots, k_{m}\right\}$ denote a generic set of lags for a given node, this data type should then consist of the following components:

(i) lags - vector containing the current set of lags, $K$, on which modeling is desired (type integer).

(ii) level - scalar specifying the level of the node in the tree (type integer).

(iii) $\Phi_{K}, \Psi_{K}$ - vectors of forward and backward model coefficient matrices; that is $\Phi_{K}=\left\{\Phi_{K}\left(k_{1}\right), \ldots, \Phi_{K}\left(k_{m}\right)\right\}$, and $\Psi_{K}=\left\{\Psi_{K}\left(k_{1}\right), \ldots, \Psi_{K}\left(k_{m}\right)\right\}$ (type real).

(iv) $U_{K}, V_{K}$ - estimates of the white noise covariance matrices for the forward and backward modeling problems (type real).

(v) $\varepsilon_{K}(t), \quad \boldsymbol{\eta}_{K}(t)$ - vectors of prediction error residuals for the forward and backward modeling problems (type real).

(vi) reg, str - pointers to the $J$ and $J^{*}$ subnodes, respectively, one level below the current level (type node, defined recursively).

The tree can be linked by recursive calls to a RECURSIVE SUBROUTINE, with level, lags, and a pointer of type node as arguments. This routine should also set a flag to signal when a particular node has been initialized (linked in the list), but the remaining components, (iii)-(vi), not yet evaluated (node unfilled). The flag, setting the first row and column entry of $U_{K}$ to zero for example, will be used by a subsequent node-filling routine. Two pointers should emanate from each node, reg pointing to $J$, and str to $J^{*}$. At level 1, these pointers should point nowhere (NULLIFY). From Figure 4, we note that both nodes at level 2 have the set $\{2\}$ as their $J^{*}$ subnode. Two copies of this subnode can be made, each linked to its appropriate parent node. This duplication of nodes can be avoided by a more complex program, since otherwise exactly $2^{m}$ nodes are created for modeling on $m$ initial lags. 


\subsection{Filling the nodes}

Once the tree with all appropriate linking pointers is in place, we will need to evaluate the remaining components, (iii)-(vi), of each node. This can be done by "walking" through the tree, following the linked list of nodes. Once again, a RECURSIVE SUBROUTINE taking a pointer as argument can be employed to achieve this, certifying first that each node has not yet been filled by checking the flag alluded to earlier.

The recursion should be implemented in such a way that the tree is walked to level 1 where the node-filling can begin. From Figure 4 , we note that this involves filling nodes $\{1\},\{2\}$, and $\{4\}$ first. With these, we can now fill nodes $\{1,3\}$ and $\{4,6\}$, at level 2 . The operation terminates at the top node, $\{1,3,7\}$, if the pointer to this node is passed as the original argument to the recursive node-filling subroutine. The pseudo-code for this phase of the implementation could therefore be:

\section{Pseudocode 2 (Fill Tree)}

call node filling routine with pointer to top node as argument while level of current node $>1$ do

call node filling routine with reg pointer as argument

if current node unfilled then fill it fi

call node filling routine with str pointer as argument

if current node unfilled then fill it fi od

if level of current node $=m$ then fill top node od

This coding will give filling-precedence to nodes at low levels that emanate from parents with respect to which they are $J$ subnodes. In example 4, this would result in the following filling order: $\{1\},\{2\}\left(J^{*}\right.$ subnode of $\{1,3\}),\{1,3\},\{4\},\{2\}\left(J^{*}\right.$ subnode of $\left.\{4,6\}\right),\{4,6\},\{1,3,7\}$.

Note that in the univariate case there is no distinction between forward and backward model parameters for the same set of lags; that is, the YW

equations give $\hat{U}_{K} \equiv \hat{V}_{K}$, and $\hat{\Phi}_{K} \equiv \hat{\Psi}_{K}$, for any set $K$. Both this and the fact that all parameters are scalars, greatly simplifies the programming task when $d=1$.

\section{Examples}

Included in Appendix C are BDT.F90 and BDT2.F90. These are, respectively, univariate and bivariate Fortran 90 programs implementing the BDT 
Algorithm. The programs utilize a few linear algebra subroutines in the International Mathematical and Statistical Library (IMSL). In this section we document how to run the programs in order to fit a particular SAR/SVAR model to a given data set, and illustrate some potential meta applications that involve repeated modeling with each of the programs in the inner loop. (We have not provided the programs for Examples 3-5, but they are available from the author upon request.)

\subsection{Example 1: Running BDT.F90}

In order to fit the model

$$
\begin{aligned}
X_{t}=\phi_{K}(1) & X_{t-1}+\phi_{K}(2) X_{t-2}+\phi_{K}(3) X_{t-3}+\phi_{K}(4) X_{t-4} \\
& +\phi_{K}(10) X_{t-10}+\phi_{K}(11) X_{t-11}+Z_{t}, \quad\left\{Z_{t}\right\} \sim \mathrm{WN}\left(0, \sigma^{2}\right),
\end{aligned}
$$

of Table 2 to the mean-corrected base 10 logarithms lynx data (lynx10.tsm in Appendix B) of Figure 2, we ran the compiled version of BDT.F90 with the following inputs at the prompt $(>)$ :

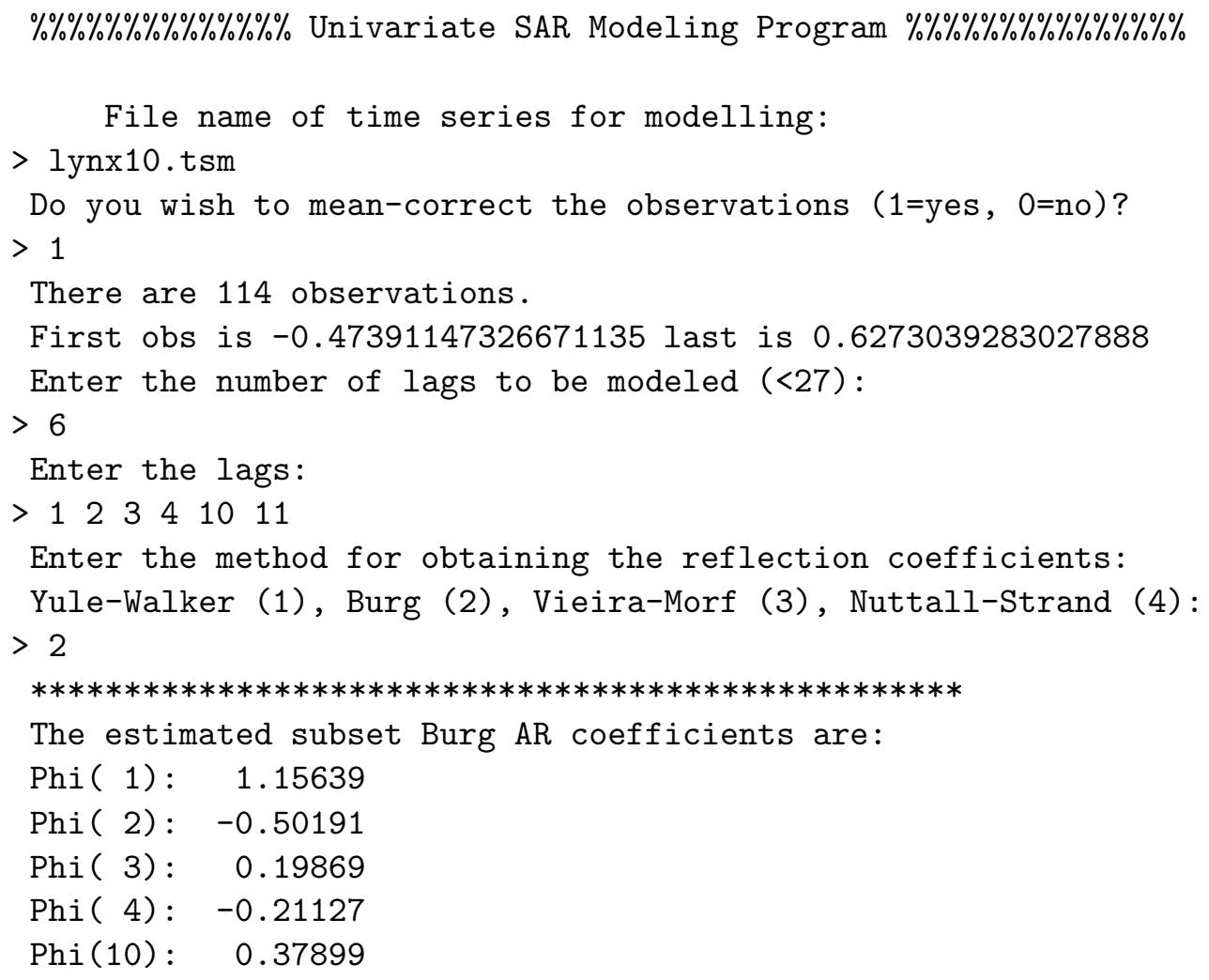


Phi(11): $\quad-0.42454$

$* * * * * * * * * * * * * * * * * * * * * * * * * * * * * * * * * * * * * * * * * * * * * * * * *$
Burg WN variance estimate : $3.61762021546651741 \mathrm{E}-2$
RSS/n WN variance estimate: $3.69130827522938937 \mathrm{E}-2$
-2 Log Likelihood (Burg) : -46.962408999128101
-2 Log Likelihood (RSS/n): -46.985742242956121
AICC (RSS $/ \mathrm{n}$ ) : - 31.929138811254461
$\% \% \% \% \% \% \% \% \% \% \% \% \% \% \% \% \% \% \% \% \% \% \% \% \% \% \% \% \% \% \% \% \% \% \% \% \% \% \% \% \% \% \% \% \% \% \% \% \% \% \% \% \% \% \% \% \% \% \%$

This gives the Burg estimators. The Yule-Walker estimates can be obtained by selecting "1" in the last step. The remaining estimators proposed by Brockwell et al. (2002), can be obtained by selecting "3" (Vieira-Morf), and "4" (Nuttall-Strand). The RSS/n is the MLE of $\sigma^{2}$ for the given set of SAR coefficient estimates, $\left\{\hat{\phi}_{K}\left(k_{1}\right), \ldots, \hat{\phi}_{K}\left(k_{m}\right)\right\}$, thus it comes as no surprise that the $-2 \log$ Likelihood based on it is never larger than the -2 Log Likelihood based on the Burg $\hat{\sigma}^{2}$. The AICC is a bias-corrected version of AIC; see Brockwell and Davis (1991).

\subsection{Example 2: Running BDT2.F90}

To fit the bivariate SVAR model

$$
\mathbf{X}_{t}=\Phi_{K}(1) \mathbf{X}_{t-1}+\Phi_{K}(3) \mathbf{X}_{t-3}+\mathbf{Z}_{t}, \quad\left\{\mathbf{Z}_{t}\right\} \sim \mathrm{WN}\left(\mathbf{0}, U_{K}\right),
$$

to the sun2.tsm data (Appendix B), we ran the compiled version of BDT2.F90 with the following inputs at the prompt $(>)$ :

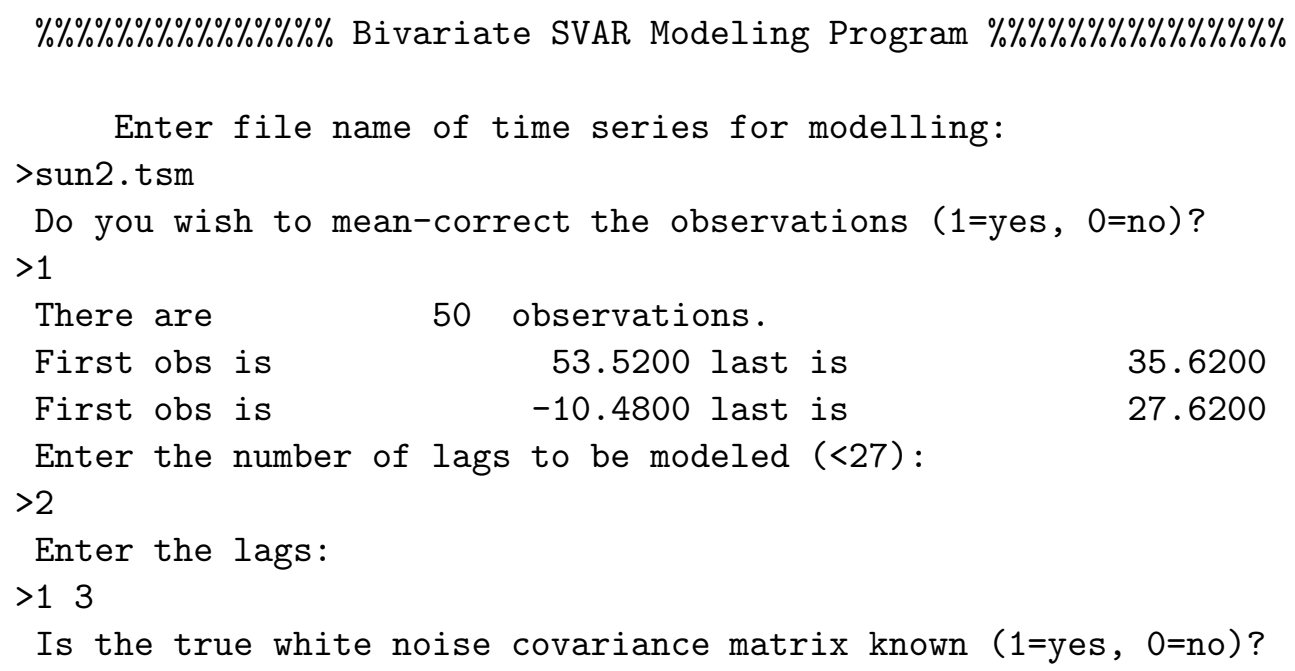




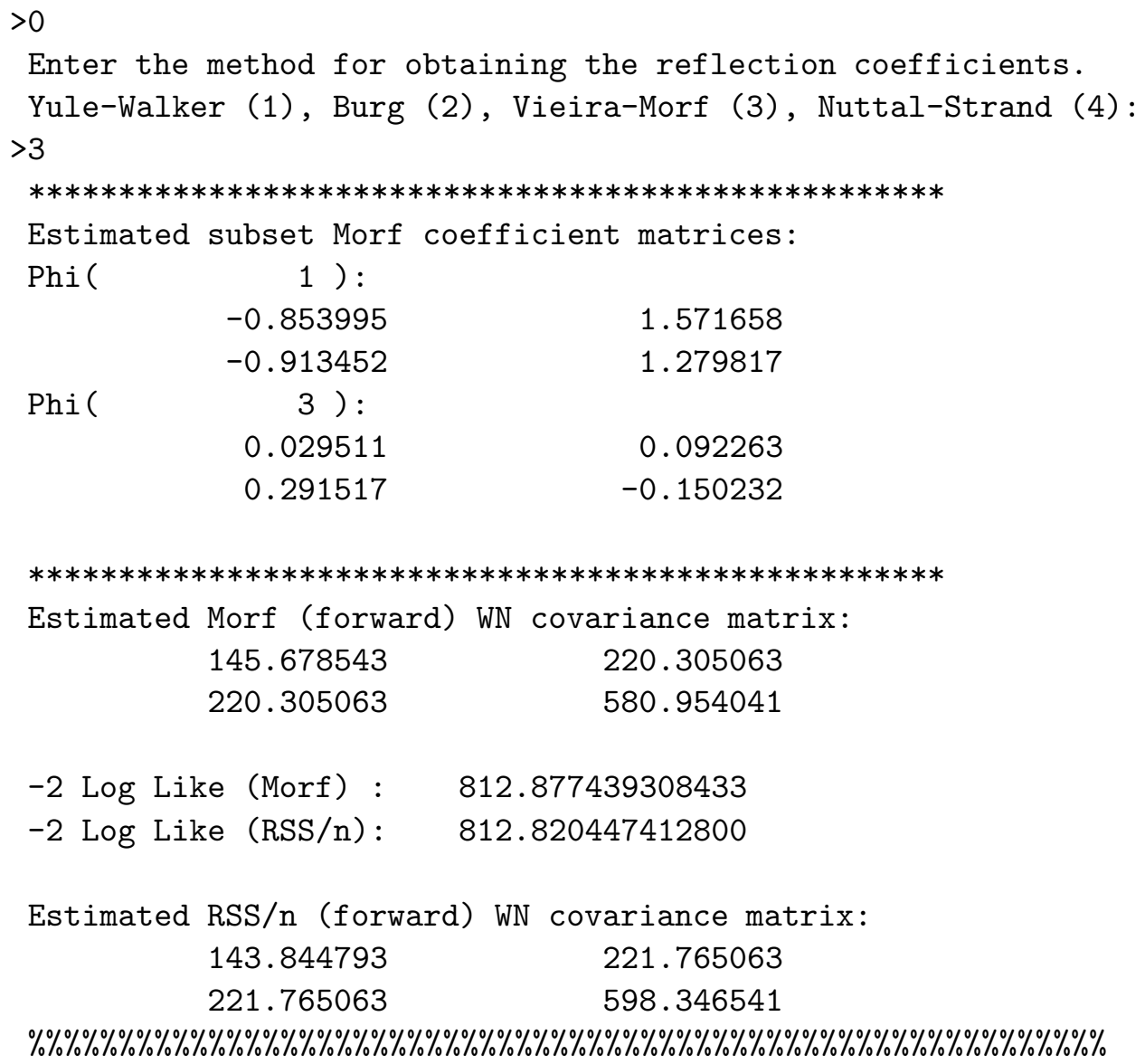

Like the univariate program, two estimates of $U_{K}$ are given: the first is method-specific (method 3, Vieira-Morf, in this case), the second, RSS/n, is the MLE of $U_{K}$ holding $\Phi_{K}(1)$ and $\Phi_{K}(3)$ fixed at their estimated values. In the multivariate case, there is no known closed form for the RSS/n estimate like there was in the univariate case (see Appendix A.6). If the data was obtained via simulation and a "1" was entered at the 5th prompt, the program would use the furnished value of $U_{K}$ as the initial guess for RSS/n in the optimizing routine. Since we entered "0", the program will use the Morf WN estimate as the initial guess.

\subsection{Example 3: Best Subset Searching}

The lynx data of Figure 2 is often cited in the literature in connection with SAR modeling. Using YW estimation and their own respective nonexhaustive search algorithms, Tong (1977), Penm and Terrell (1982), Zhang 
and Terrell (1997), and others, identify a $\operatorname{SAR}(1,2,4,10,11)$, i.e. $K=\{1,2,4,10,11\}$, as the best SAR model according to a variety of information criteria. It is important to realize that some of these search methods are non-exhaustive and statistical in nature, and will therefore not guarantee a correct identification with certainty.

Using the BDT Algorithm, we performed an exhaustive search for the minimum AICC SAR model, for the mean-corrected base 10 logarithms of the lynx data. Letting $p$ denote the maximum lag considered in the search (meaning that $2^{p}$ models had to be checked), we considered $p=4,8$, and 12 , in turn. This set of searches was performed for each of the YW and Burg estimation methods. The number of subsets out of the $2^{p}$ that resulted in non-causal fitted models, as well as the corresponding computational (CPU) times taken by each search, were recorded. The AICC (RSS/n) of the best SAR model was also computed.

Table 1: Results of best (minimum AICC) SAR model search for the meancorrected base 10 logarithms of the Canadian lynx data of Figure 2.

\begin{tabular}{lc|c|c|c|c}
\hline \hline $\begin{array}{l}\text { Estimation } \\
\text { method }\end{array}$ & $p$ & $\begin{array}{c}\text { Lags in } \\
\text { best subset }\end{array}$ & $\begin{array}{c}\text { AICC of } \\
\text { best model }\end{array}$ & $\begin{array}{c}\text { Prop. of non } \\
\text { causal models }\end{array}$ & $\begin{array}{c}\text { CPU time } \\
\text { (secs) }\end{array}$ \\
\hline \multirow{3}{*}{ YW } & 4 & $1,2,4$ & -9.89 & $3 / 16$ & 1.2 \\
& 8 & $1,2,4,8$ & -16.17 & $78 / 256$ & 21.3 \\
& 12 & $1,2,4,10,11$ & -31.80 & $1392 / 4096$ & 637.2 \\
& & & & & \\
Burg & 4 & $1,2,4$ & -10.08 & $3 / 16$ & 1.8 \\
& 12 & $1,2,4,8$ & -16.27 & $81 / 256$ & 29.6 \\
& $12,3,4,10,11$ & -31.93 & $1489 / 4096$ & 678.7 \\
\hline \hline
\end{tabular}

The results are summarized in Table 1 . The best SAR model with maximum lag 12 found by the YW method, coincides with that identified by other researchers as discussed above; but that arrived at by the new subset Burg method, adds lag 3, and has a slightly lower value of AICC. Note also that the proportion of subsets resulting in non-causal fitted models (meaning that a SAR model with these lags is inappropriate for the data), remained steady at approximately $1 / 3$ across all searches. At the same value of $p$, CPU times for Burg are slightly higher than those for YW, both growing exponentially with $p$. The computations were carried out on a Sun Enter- 
prise 450 unix server, equipped with about $4 \mathrm{G}$ of memory. The most severe limiting factor in this type of computation is available memory, since at least $2^{p}$ pointers have to be allocated.

In Table 2, we present the parameter estimates of the best SAR model identified by each respective method when $p=12$. The constrained ML estimates were obtained via the ITSM2000 package (Brockwell and Davis, 2002), starting with the Burg estimates.

Table 2: Best SAR models fitted to the mean-corrected base 10 logarithms of the Canadian lynx data, as identified by each respective method. The Maximum Likelihood estimates were obtained by starting with the Burg estimates, and constraining the ML search to the same SAR lags.

\begin{tabular}{||c||c|r|c||}
\hline \hline \multicolumn{1}{||c||}{ Parameter } & \multicolumn{3}{c||}{ Estimates by Method } \\
\cline { 2 - 4 } & Maximum Likelihood & \multicolumn{1}{c||}{ Burg } & Yule-Walker \\
\hline \hline$\phi_{K}(1)$ & 1.148 & 1.156 & 1.094 \\
$\phi_{K}(2)$ & -0.502 & -0.502 & -0.357 \\
$\phi_{K}(3)$ & 0.199 & 0.199 & \\
$\phi_{K}(4)$ & -0.217 & -0.211 & -0.127 \\
$\phi_{K}(10)$ & 0.351 & 0.379 & 0.324 \\
$\phi_{K}(11)$ & -0.401 & -0.425 & -0.362 \\
$\sigma^{2}$ & 0.037 & 0.037 & 0.038 \\
AICC & -32.22 & -31.93 & -31.80 \\
\hline \hline
\end{tabular}

\subsection{Examples 4 and 5: Adaptive Behavior}

In these simulated bivariate examples, we illustrate the component-wise convergence of the Burg and YW estimates to their true values, as a function of observation number or time. This adaptive behavior is important in online applications where it is desirable to monitor the convergence of the estimates, particularly when observation number is still low. Note however that the BDT Algorithm is recursive in the model order, not observation number, and is therefore not truly adaptive in that sense. The entire Algorithm needs to be re-run from scratch whenever a new observation becomes available.

The characteristic polynomial of SVAR model (1) is

$$
P(z)=\operatorname{det}\left[I_{d}-\Phi_{K}\left(k_{1}\right) z^{k_{1}}-\cdots-\Phi_{K}\left(k_{m}\right) z^{k_{m}}\right] .
$$


The model is causal if the zeroes of its characteristic polynomial are all greater than one in magnitude. It is well-known that in the univariate fullset case, the YW estimators can be severely biased if the roots of the AR characteristic polynomial are close to the unit circle (quasi-non-stationarity). To allow for the expected dependence of performance on the location of the zeroes of $P(z)$, we considered causal models with different configurations of these zeroes. A total of 250 observations were sequentially simulated from the basic $\operatorname{SVAR}(2)$ model,

$$
\mathbf{X}_{t}=\Phi \mathbf{X}_{t-2}+\mathbf{Z}_{t} \equiv\left[\begin{array}{ll}
\Phi_{11} & \Phi_{12} \\
\Phi_{21} & \Phi_{22}
\end{array}\right] \mathbf{X}_{t-2}+\mathbf{Z}_{t},, \quad \mathbf{Z}_{t} \sim \mathrm{N}_{2}\left(\mathbf{0}, I_{2}\right)
$$

and we started estimation at observation number 10 .

\section{Example 4}

$$
\Phi=\left[\begin{array}{ll}
0.547 & -0.300 \\
0.700 & -0.457
\end{array}\right], \quad P(z)=\left(1-0.25 z^{2}\right)\left(1+0.16 z^{2}\right)
$$

with roots of characteristic polynomial: $\pm 2, \pm 2.5 i$.

\section{Example 5}

$$
\Phi=\left[\begin{array}{cc}
1.414 & -0.300 \\
0.700 & 0.497
\end{array}\right], \quad P(z)=\left(1-0.98^{2} z^{2}\right)\left(1-0.95^{2} z^{2}\right),
$$

with roots of characteristic polynomial: $\pm 1.02, \pm 1.03$.

The results are displayed in Figure 5, where we plot the componentwise departures of the estimated SVAR coefficient matrices from their true values, $\Phi_{i j}-\hat{\Phi}_{i j}, i, j=1,2$, as a function of observation number. The pattern of convergence between the two methods is similar in Example 4, but dramatically different in Example 5. Although based on a single simulated realization presented only to illustrate a meta application of the bivariate BDT Algorithm, this phenomenon is nevertheless consistent with what has been noted about the behavior of YW versus Burg in quasi-non-stationary modeling. Since both estimators and the MLE all have the same asymptotic distribution, there is little cause for concern with large samples; it is with small samples that one should exercise caution when selecting an estimation method. The more extensive analysis by Brockwell et al. (2002), suggests that Burg is in general a better estimator than YW. 
Figure 5: Departures of the 4 estimated components of the SVAR coefficient matrix from their true values, by method, for the simulated realizations of Examples 4 and 5 .

Ex 4: YW Method

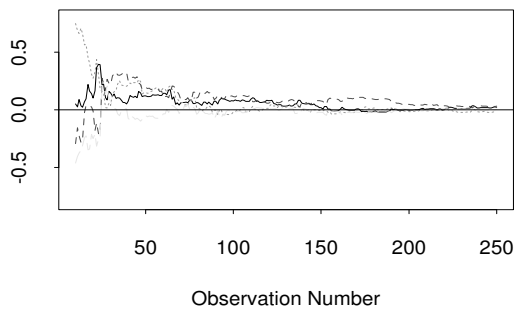

Ex 4: Burg Method

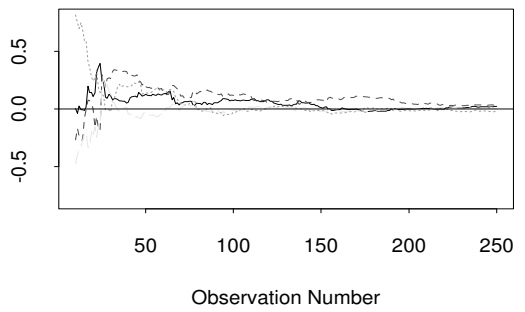

Ex 5: YW Method

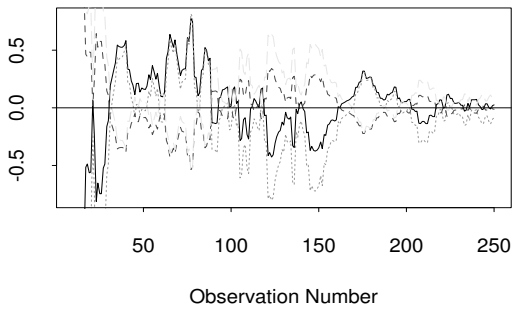

Ex 5: Burg Method

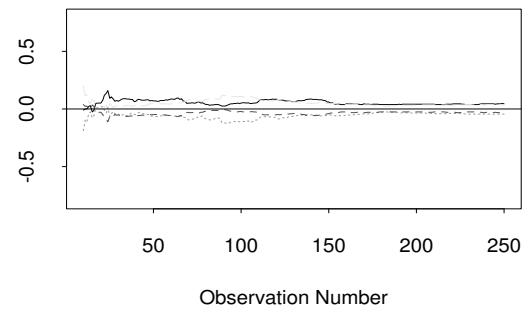

\section{Conclusion}

We have discussed the popularity of multivariate subset autoregressive models, and highlighted the importance of fast, simple, and efficient methods for the estimation of their parameters. One such set of estimators is obtained via the classical Yule-Walker method-of-moments, which we have presented as the solution to a system of simultaneous linear matrix equations. A recently introduced more general estimation method, the BDT Algorithm, is recursive in the order of the fitted model, thus avoiding the (potentially large) matrix inversions required in solving the Yule-Walker equations. By suitably modifying the reflection coefficient calculation, this Algorithm can produce a variety of estimators with different finite sample properties, among them Yule-Walker. We have illustrated the recursive structure of this Algorithm, 
and discussed its implementation in a high-level programming language like Fortran 90. The speed of the Algorithm was assessed in finding a best subset model for the Canadian lynx data, and shown, in problems of moderate size, to be a feasible alternative to non-exhaustive search techniques which do not guarantee correct subset identification. We concluded with two simulated bivariate examples that illustrate the adaptive performance of the YuleWalker and Burg estimators, implemented via the BDT Algorithm. We find that the Burg estimates tend to stabilize more quickly than Yule-Walker, and are far less affected by proximity of the model to non-stationarity.

\section{Acknowledgements}

The author would like to acknowledge NSF for partial support of this research through grant number DMS-9972015, and the invaluable suggestions provided by the referee who also reviewed the code.

\section{References}

[1] Brockwell, P.J., Dahlhaus, R., and Trindade, A.A. (2002), "Modified Burg Algorithms for Multivariate Subset Autoregression", Technical Report 2002-015, Department of Statistics, University of Florida.

[2] Brockwell P.J., and Davis R.A. (1991), Time Series: Theory and Methods (2nd ed.), New York: Springer-Verlag.

[3] Brockwell P.J., and Davis R.A. (2002), Introduction to Time Series and Forecasting, Second Ed., New York: Springer-Verlag.

[4] Burg, J.P. (1968), "A New Analysis Technique for Time Series Data", in Modern Spectrum Analysis, (1978), D.G. Childers (ed.), NATO Advanced Study Institute of Signal Processing with emphasis on Underwater Acoustics, New York: IEEE Press.

[5] Godsill, S.J., and Rayner P.J.W. (1998), Digital Audio Restoration: A Statistical Model-Based Approach, Berlin: Springer.

[6] Hooke, R., and Jeeves T. (1961), "A direct search solution of numerical and statistical problems", Journal of Association for Computing Machinery, 8, 212-229. 
[7] McClave, J. (1975), "Subset Autoregression", Technometrics, 17, 213-220.

[8] Penm, J.H. and Terrell R.D. (1982), "On the Recursive Fitting of Subset Autoregressions", Journal of Time Series Analysis, 3, 43-59.

[9] Sarkar, A. and Sharma, K.M.S. (1997), "An Approach to Direct Selection of Best Subset AR Model", Journal of Statistical Computation and Simulation, 56, 273-291.

[10] Tong, H. (1977), "Some comments on the Canadian lynx data", Journal of the Royal Statistical Society, Series A, 140, 432-436.

[11] Zhang, X. and Terrell, R.D. (1997), "Projection Modulus: A New Direction for Selecting Best Subset Autoregressive Models", Journal of Time Series Analysis, 18, 195-212.

\section{A Description of Principal Program Subroutines}

As already stated, the core of the subset modeling programs Burg and Burg2 is the globally visible MODULE Tree, with SUBROUTINE Make_Tree its driving subroutine. In this section, we will provide a brief description of the essential functions of its main constituent subroutines.

\section{A.1 Build_Node_Tree}

This is a RECURSIVE SUBROUTINE that initializes the tree of nodes by allocating pointers to and from nodes. It takes on the level, lags, and a pointer of type node as arguments. It begins execution at the unique node of level $m$ (top_node), creating pointers to the $J$ and $J^{*}$ subnodes (this_node\%reg and this_node\%str, respectively). Following these pointers to level $m-1$, Build_Node_Tree subsequently allocates pointers to the subnodes in level $m-2$. It achieves this by calling itself with the appropriate arguments:level should be the current level minus one, and pointers this_node\%reg and this_node\%str. The procedure is repeated, always following pointer this_node\%reg before this_node\%str, until level 1 is reached. At this point, the two pointers are initialized and made to point nowhere (NULLIFIED). By the order of precedence inherent in it, the routine then backs up one level and proceeds to follow pointer this_node\%str to the "dead end" at level 1. 
In this fashion, the tree is initialized from left $(J)$ to right $\left(J^{*}\right)$, with the pointer to the subnode $J^{*}$ of the rightmost node being allocated last. If we refer back to figure 4 , the nodes for the tree of this example will be initialized in the following order:

$$
\{1,3,7\} \rightarrow\{1,3\} \rightarrow\{1\} \rightarrow\{2\} \rightarrow\{4,6\} \rightarrow\{4\} \rightarrow\{2\} .
$$

In order for subsequent routines to identify an initialized but unfilled (constituents of node empty) node, Build_Node_Tree will set this_node\%v (this_node $\% \mathrm{vf} \% \operatorname{mat}(1,1)$ in BDT2.F90) to zero, upon allocation of pointers.

\section{A.2 Fill_Tree}

A RECURSIVE SUBROUTINE, taking on a pointer of type node as argument. Its function is to traverse the now initialized tree, and using the flag for an unfilled node, fill it by calling Fill_Node.

\section{A.3 Fill_Node}

A RECURSIVE SUBROUTINE, called by Fill_Tree, whose function is to fill the particular node that its pointer argument points to. It is in this routine that the BDT Algorithm proper is applied, modifying the reflection coefficient calculation according to the selected method. Care must be taken when calculating the forward and backward prediction errors, $\varepsilon_{K}(t)$ and $\boldsymbol{\eta}_{K}(t)$, before termination of the routine. Each should be calculated over a sufficiently large range of $t$ values $\left(1 \leq t \leq n+k_{m}\right.$ for Yule-Walker, and $1+k_{m} \leq t \leq n$ for the remaining methods), since subsequent nodes may use them.

\section{A.4 Print_Node_Tree}

With its pointer argument, the RECURSIVE SUBROUTINE Print_Node_Tree will traverse the now completed tree of nodes, and proceed to print the estimated coefficients and white noise variance stored in each node.

\section{A.5 Causal_Check}

This routine is needed in the bivariate program only, in order to ensure the obtained VAR model is causal before proceeding with the likelihood calculations. In the univariate program, this function is performed within 
the likelihood calculation routine itself. The strategy is to use the state space representation to write a $\operatorname{VAR}(p)$ as a $\operatorname{VAR}(1)$, as follows:

Random vectors $\left\{\mathbf{X}_{t}, \ldots, \mathbf{X}_{t-k_{m}}\right\}$ from model (1), will satisfy the relationships

$$
\left[\begin{array}{c}
\mathbf{X}_{t} \\
\mathbf{X}_{t-1} \\
\mathbf{X}_{t-2} \\
\vdots \\
\mathbf{X}_{t-k_{m}+1}
\end{array}\right]=\left[\begin{array}{ccccccc}
0 & 0 & \cdots & 0 & k_{1} & \cdots & k_{m} \\
I_{d} & 0 & \cdots & \cdots & \cdots & \cdots & 0 \\
0 & I_{d} & & & & & 0 \\
& & \ddots & & & & \\
\vdots & & & \ddots & & & \vdots \\
& & & & \ddots & & \\
0 & & & \cdots & & I_{d} & 0
\end{array}\right]\left[\begin{array}{c}
\mathbf{X}_{t-1} \\
\mathbf{X}_{t-2} \\
\mathbf{X}_{t-3} \\
\\
\vdots \\
\\
\\
\\
\mathbf{X}_{t-k_{m}}
\end{array}\right]+\left[\begin{array}{c}
\mathbf{Z}_{t} \\
\mathbf{0} \\
\mathbf{0} \\
\vdots \\
\\
\\
\\
\end{array}\right]
$$

which can be written in the compact form

$$
\underbrace{\mathbf{Y}_{t}}_{\left(d k_{m} \times 1\right)}=\underbrace{A}_{\left(d k_{m} \times d k_{m}\right)} \underbrace{\mathbf{Y}_{t-1}}_{\left(d k_{m} \times 1\right)}+\underbrace{\mathbf{W}_{t}}_{\left(d k_{m} \times 1\right)} .
$$

In block matrix form, vectors $\mathbf{Y}_{t}$ and $\mathbf{W}_{t}$ have length $k_{m}$, while the square matrix $A$ has dimension $k_{m}$. Note that the only nonzero entries of the first block matrix row of $A$ are $\left\{\Phi_{K}\left(k_{1}\right), \Phi_{K}\left(k_{2}\right), \ldots, \Phi_{K}\left(k_{m-1)}, \Phi_{K}\left(k_{m}\right)\right\}\right.$, occurring at block matrix column numbers $\left\{k_{1}, k_{2}, \ldots, k_{m-1}, k_{m}\right\}$, respectively. The covariance matrix of $\mathbf{W}_{t}$ is

$$
\Sigma_{W}=\mathbf{E}\left[\begin{array}{c}
\mathbf{Z}_{t} \\
\mathbf{0} \\
\vdots \\
\mathbf{0}
\end{array}\right]\left[\mathbf{Z}_{t}^{\prime}, \mathbf{0}^{\prime}, \ldots, \mathbf{0}^{\prime}\right]=\left[\begin{array}{cccc}
\Sigma & 0 & \cdots & 0 \\
0 & 0 & \cdots & 0 \\
\vdots & & \ddots & \vdots \\
0 & \cdots & \cdots & 0
\end{array}\right]
$$

where we use $\Sigma$ in place of $U_{K}$. (28) is now a $\operatorname{VAR}(1)$ of dimension $d k_{m}$, and its causality (and thus that of the original process) can be assessed by determining if all eigenvalues of $A$ are less than 1 in absolute value.

\section{A.6 Likelihood/Approx_Likelihood}

In the univariate program, we compute the exact likelihood in SUBROUTINE Likelihood. The only sizeable difficulty is in evaluating the model autocovariances $\gamma(0), \ldots, \gamma\left(k_{m}\right)$, accomplished by inverting the Yule-Walker equations. The $-2 \log$ likelihood, $\mathcal{L}\left(\phi_{K}, \sigma^{2}\right)$, for the data $\mathbf{x}_{1}, \ldots, \mathbf{x}_{n}$, is 
then evaluated via the Innovations Algorithm (Brockwell and Davis, 1991, prop. 5.2.2, and equation 8.7.4):

$$
\mathcal{L}\left(\phi_{K}, \sigma^{2}\right)=n \log \left(2 \pi \sigma^{2}\right)+\sum_{t=1}^{n} \log \left(r_{t-1}\right)+\frac{1}{\sigma^{2}} \sum_{t=1}^{n}\left(\mathbf{x}_{t}-\hat{\mathbf{x}}_{t}\right)^{2} / r_{t-1} .
$$

In the bivariate program, SUBROUTINE Likelihood uses the same approach to compute the likelihood, i.e. the Multivariate Innovations Algorithm (Brockwell and Davis, 1991, prop. 11.4.2, and equation 11.5.5):

$$
\mathcal{L}\left(\Phi_{K}, \Sigma\right)=n d \log (2 \pi)+\sum_{t=1}^{n} \log \left|V_{t-1}\right|+\sum_{t=1}^{n}\left(\mathbf{X}_{t}-\hat{\mathbf{X}}_{t}\right)^{\prime} V_{t-1}^{-1}\left(\mathbf{X}_{t}-\hat{\mathbf{X}}_{t}\right)
$$

Computing the model autocovariance matrices, $\Gamma\left(1-k_{m}\right), \ldots, \Gamma(0), \ldots, \Gamma\left(k_{m}-\right.$ $1)$, is a much more formidable task here, but this can be accomplished via the state space formulation of the previous subsection. Transforming the $\operatorname{SVAR}(K)$ to the $\operatorname{VAR}(1)$ of equation (28), gives the following solution for the autocovariances $\Gamma_{Y}(\cdot)$ of the process $\left\{\mathbf{Y}_{t}\right\}$ :

$$
\Gamma_{Y}(h)=\left\{\begin{array}{ll}
A \Gamma_{Y}(h) A^{\prime}+\Sigma_{W}, & h=0 \\
A \Gamma_{Y}(h-1), & h>0
\end{array},\right.
$$

whence we obtain

$$
\operatorname{vec}\left(\Gamma_{Y}(0)\right)=\left[I_{d^{2} k_{m}^{2}}-A \otimes A\right]^{-1} \operatorname{vec}\left(\Sigma_{W}\right) .
$$

The required autocovariance matrices can be found in the first block row and column of the $\left(k_{m} \times k_{m}\right)$ block matrix $\Gamma_{Y}(0)$, since

$$
\underbrace{\Gamma_{Y}(0)}_{\left(d k_{m} \times d k_{m}\right)}=\left[\begin{array}{cccc}
\Gamma(0) & \Gamma(1) & \cdots & \Gamma\left(k_{m}-1\right) \\
\Gamma(-1) & \Gamma(0) & \cdots & \Gamma\left(k_{m}-2\right) \\
\vdots & & \ddots & \vdots \\
\Gamma\left(1-k_{m}\right) & \cdots & \Gamma(-1) & \Gamma(0)
\end{array}\right] .
$$

Due to the computational intensity involved in finding $\Gamma_{Y}(\cdot)$ however, the bivariate routine Likelihood is extremely slow. We opt instead to approximate the autocovariances via the causal representation

$$
\Gamma(h)=\sum_{j=0}^{\infty} \Psi_{h+j} \Sigma \Psi_{j}^{\prime},
$$


truncating the summation at 100 terms, and computing the likelihood via (2). This "approximate likelihood", is computed in SUBROUTINE Obj Fun. SUBROUTINE Approx_Likelihood not only calls Obj Fun in order to compute this approximate likelihood for $\Sigma_{A L}$, but also searches for the white noise covariance matrix that maximizes the likelihood for the given VAR coefficient matrices $\left(\Sigma_{M L}\right)$. It does so by using $\Sigma_{A L}$ as an initial guess, and by repeated calls to SUBROUTINE Hooke, which employs a direct search algorithm to locate the global minimum of an objective function of several variables (Hooke and Jeeves, 1961).

\section{B Data Sets}

\section{B.1 lynx10.tsm}

2.42975228000241

2.50650503240487

2.76715586608218

2.94001815500766

3.16879202031418

3. 45040308615537

3. 59417147911491

3. 77400573025821

3. 69460519893357

3. 4111144185509

2.71850168886727

1. 99122607569249

2. 26481782300954

2. 4456042032736

2. 61172330800734

3. 35888620440587

3. 42894429003557

3. 53262700122889

3. 2610248339924

2. 61172330800734

2. 17897694729317

1. 65321251377534

1. 83250891270624

2.32837960343874

2. 73719264270474

3. 01410032151962 
3.32817566143832
3.40414924920969
2.98091193777684
2.55750720190566
2.57634135020579
2.35218251811136
2.55630250076729
2.86391737695786
3.2143138974244
3.43536650661266
3.45803319249651
3.32613095671079
2.83505610172012
2.47567118832443
2.37291200297011
2.38916608436453
2.7419390777292
3.21031851982623
3.51995918075207
3.82743389540078
3.62879748556671
2.83695673705955
2.40654018043395
2.67486114073781
2.55388302664387
2.89431606268444
3.20248831706009
3.22427401429426
3.35237549500052
3.15411952551585
2.87852179550121
2.47567118832443
2.30319605742049
2.35983548233989
2.67117284271508
2.8668778143375
3.31005573775089
3.44886084560744
3.64650175003161
3.39984671271292 
2.58994960132571
1.86332286012046
1.5910646070265
1.69019608002851
1.77085201164214
2.27415784926368
2.57634135020579
3.11126251365907
3.60541279815305
3.5434471800817
2.76863810124761
2.02118929906994
2.1846914308176
2.58771096501891
2.87966920563205
3.11627558758054
3.53970323894783
3.84453930212901
3.80023578932735
3.57909732655264
3.26387267686522
2.53781909507327
2.58206336291171
2.90741136077459
3.14238946611884
3.4334497937616
3.57978359661681
3.4900990050633
3.47494433546539
3.57863920996807
2.82865989653532
1.90848501887865
1.90308998699194
2.03342375548695
2.35983548233989
2.60097289568675
3.05384642685225
3.3859635706007
3.55315454816963
3.46760810558363 
3.18667386749974

2.72345567203519

2.68574173860226

2.8208579894397

3

3. 20139712432045

3.42439155441028

3. 53096768157191

\section{B.2 sun2.tsm}

101.000000000000000

66.000000000000000

31.000000000000000

20.000000000000000

154.000000000000000

85.000000000000000

38.000000000000000

10.000000000000000

83.000000000000000

131.000000000000000

90.000000000000000

60.000000000000000

41.000000000000000

16.000000000000000

4.000000000000000

14.000000000000000

45.000000000000000

48.000000000000000

28.000000000000000

8.000000000000000

$0.000000000000000 \mathrm{E}+000$

5.000000000000000

14.000000000000000

46.000000000000000

30.000000000000000

16.000000000000000

4.000000000000000

8.000000000000000

36.000000000000000
82.000000000000000

35.000000000000000

7.000000000000000

92.000000000000000

125.000000000000000

68.000000000000000

23. 000000000000000

24.000000000000000

132.000000000000000

118.000000000000000

67.000000000000000

47.000000000000000

21.000000000000000

6.000000000000000

7.000000000000000

34.000000000000000

43.000000000000000

42.000000000000000

10.000000000000000

2.000000000000000

1.000000000000000

12.000000000000000

35.000000000000000

41.000000000000000

24.000000000000000

7.000000000000000

2.000000000000000

17.000000000000000

50.000000000000000 


$\begin{array}{rr}62.000000000000000 & 67.000000000000000 \\ 71.000000000000000 & 48.000000000000000 \\ 28.000000000000000 & 8.000000000000000 \\ 13.000000000000000 & 57.000000000000000 \\ 122.000000000000000 & 138.000000000000000 \\ 103.000000000000000 & 86.000000000000000 \\ 63.000000000000000 & 37.000000000000000 \\ 24.000000000000000 & 11.000000000000000 \\ 15.000000000000000 & 40.000000000000000 \\ 62.000000000000000 & 98.000000000000000 \\ 124.000000000000000 & 96.000000000000000 \\ 66.000000000000000 & 64.000000000000000 \\ 54.000000000000000 & 39.000000000000000 \\ 21.000000000000000 & 7.000000000000000 \\ 4.000000000000000 & 23.000000000000000 \\ 55.000000000000000 & 94.000000000000000 \\ 96.000000000000000 & 77.000000000000000 \\ 59.000000000000000 & 44.000000000000000 \\ 47.000000000000000 & 30.000000000000000 \\ 16.000000000000000 & 7.000000000000000 \\ 37.000000000000000 & 74.000000000000000\end{array}$

\section{Coded Versions of the BDT Algorithm}

\section{C.1 Code for BDT.F90}

MODULE tree

! Here we define the data type NODE which will contain TYPE node

INTEGER $:$ : level ! level in tree: top=m, bottom=1

INTEGER :: lags(26) ! lags for node are stored here

DOUBLE PRECISION $::$ phi(26) ! coefficients for node

DOUBLE PRECISION $:: \mathrm{V}$ ! MSE (white noise) for node

DOUBLE PRECISION $::$ eps $(1: 10100)$ ! the epsilons for the node

DOUBLE PRECISION $:$ : eta $(-99: 10000)$ ! the etas for the node

TYPE (node), POINTER : : reg, star ! pointers to the regular and

END TYPE node

! starred subnodes one level down

! These will contain the end results 


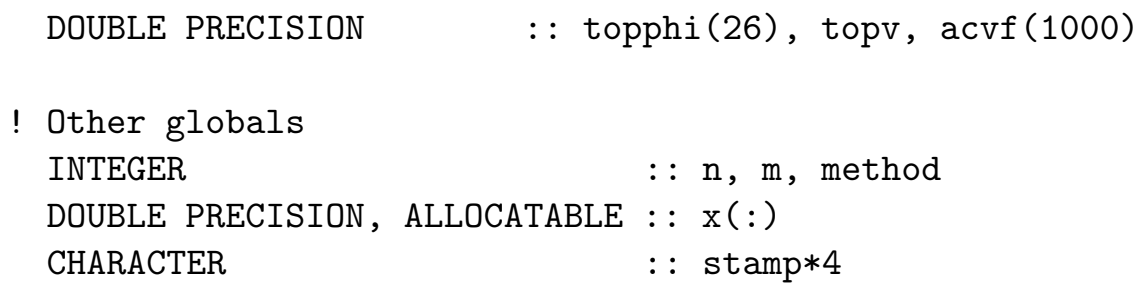

\section{CONTAINS}

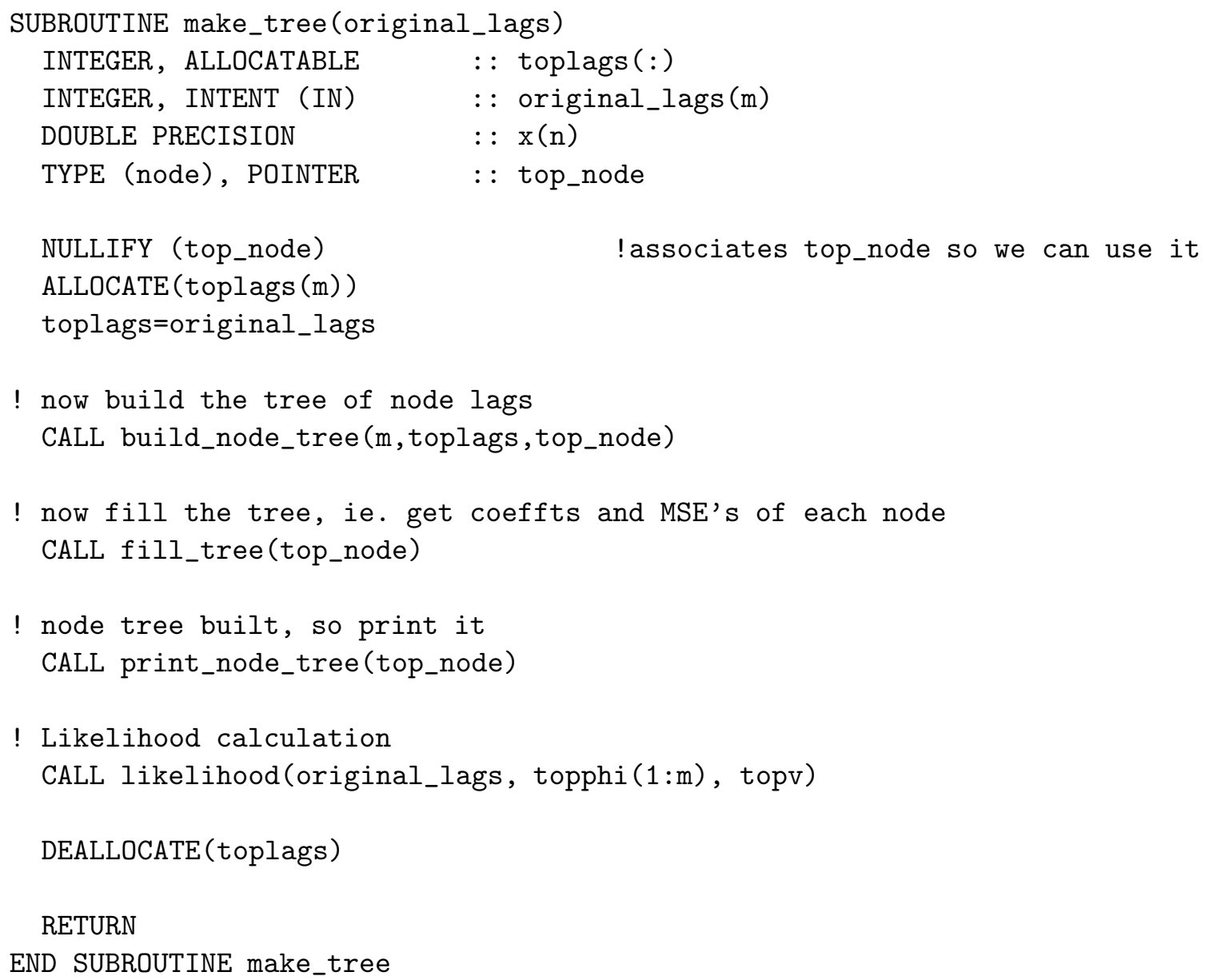




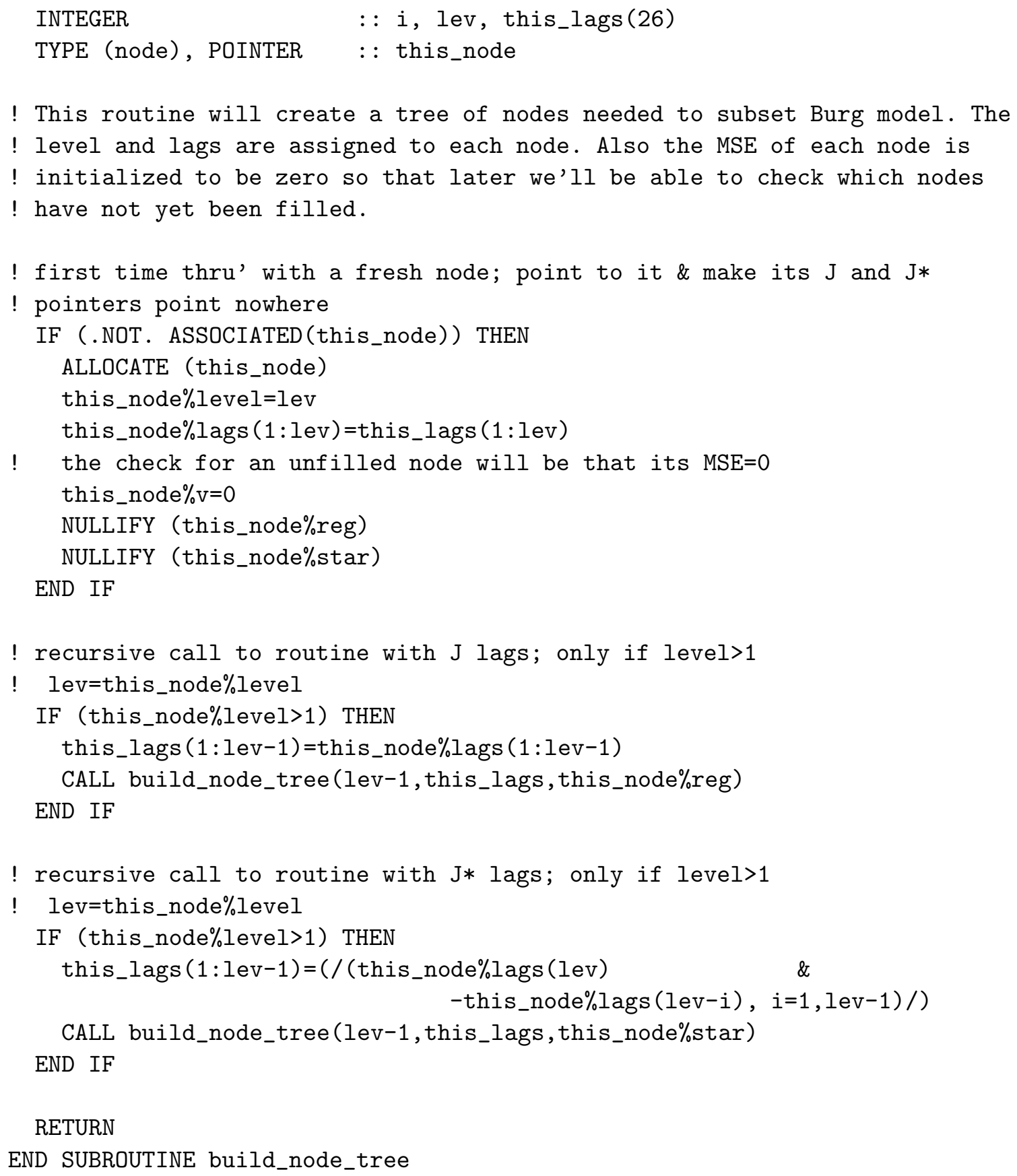




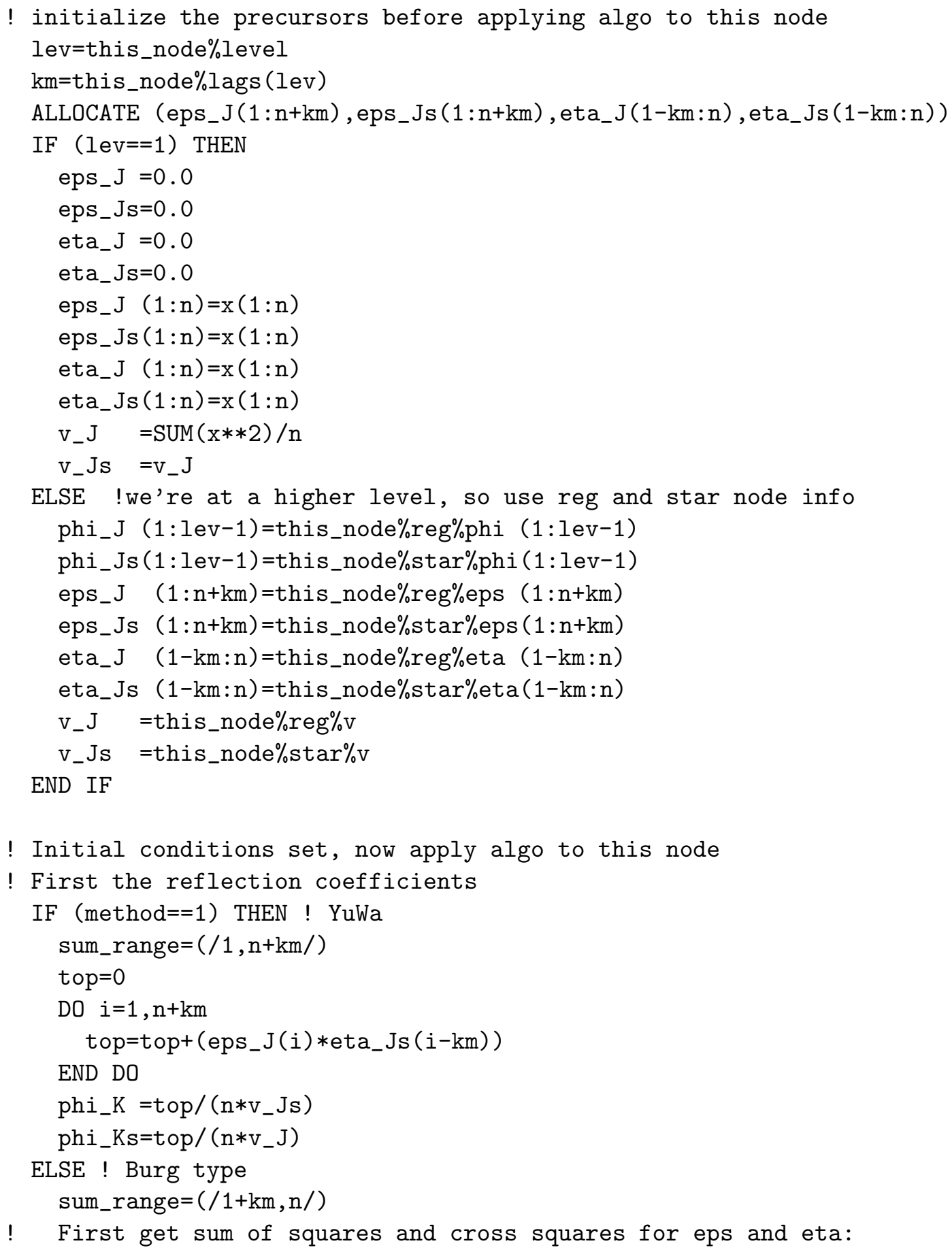




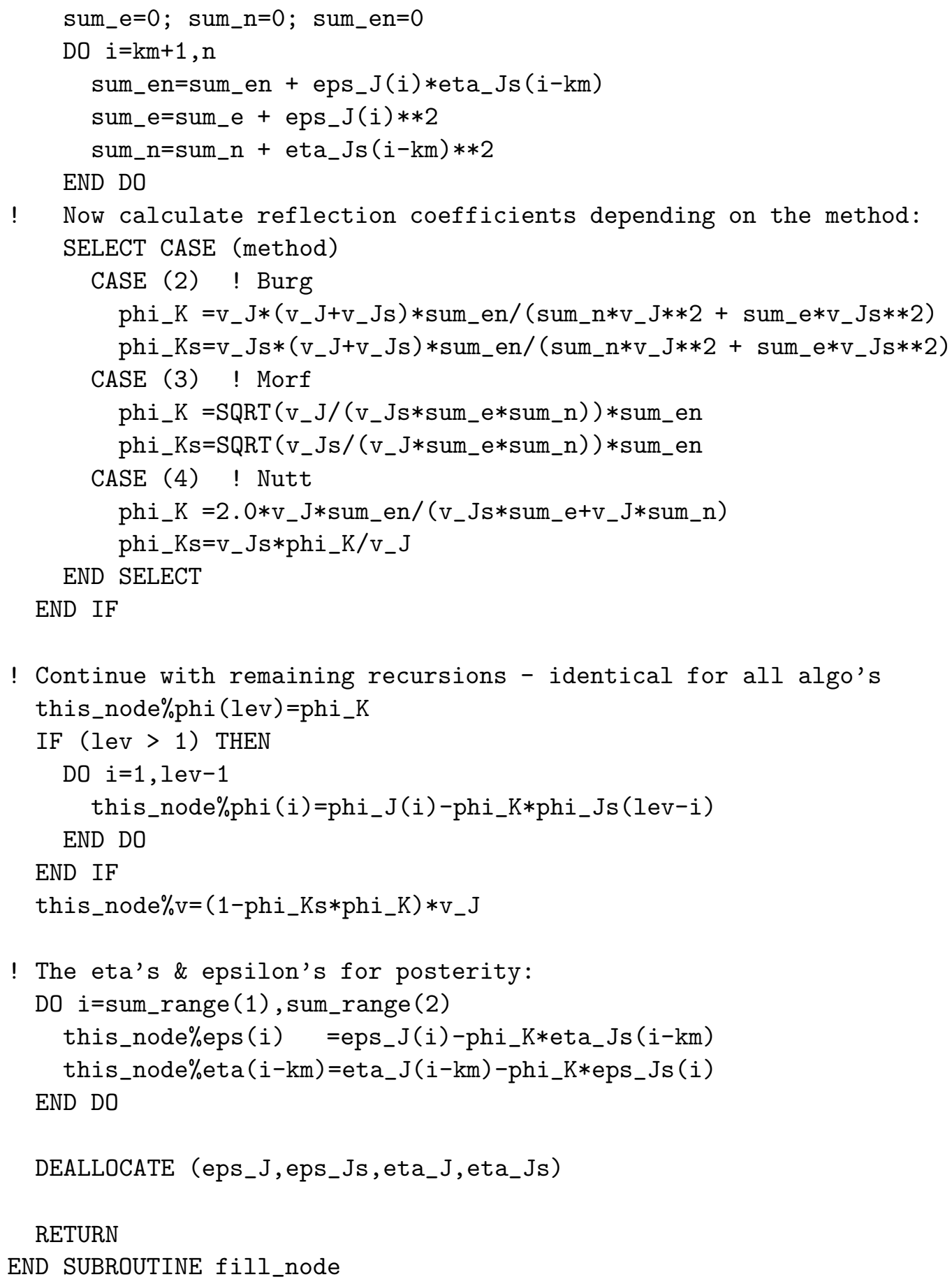




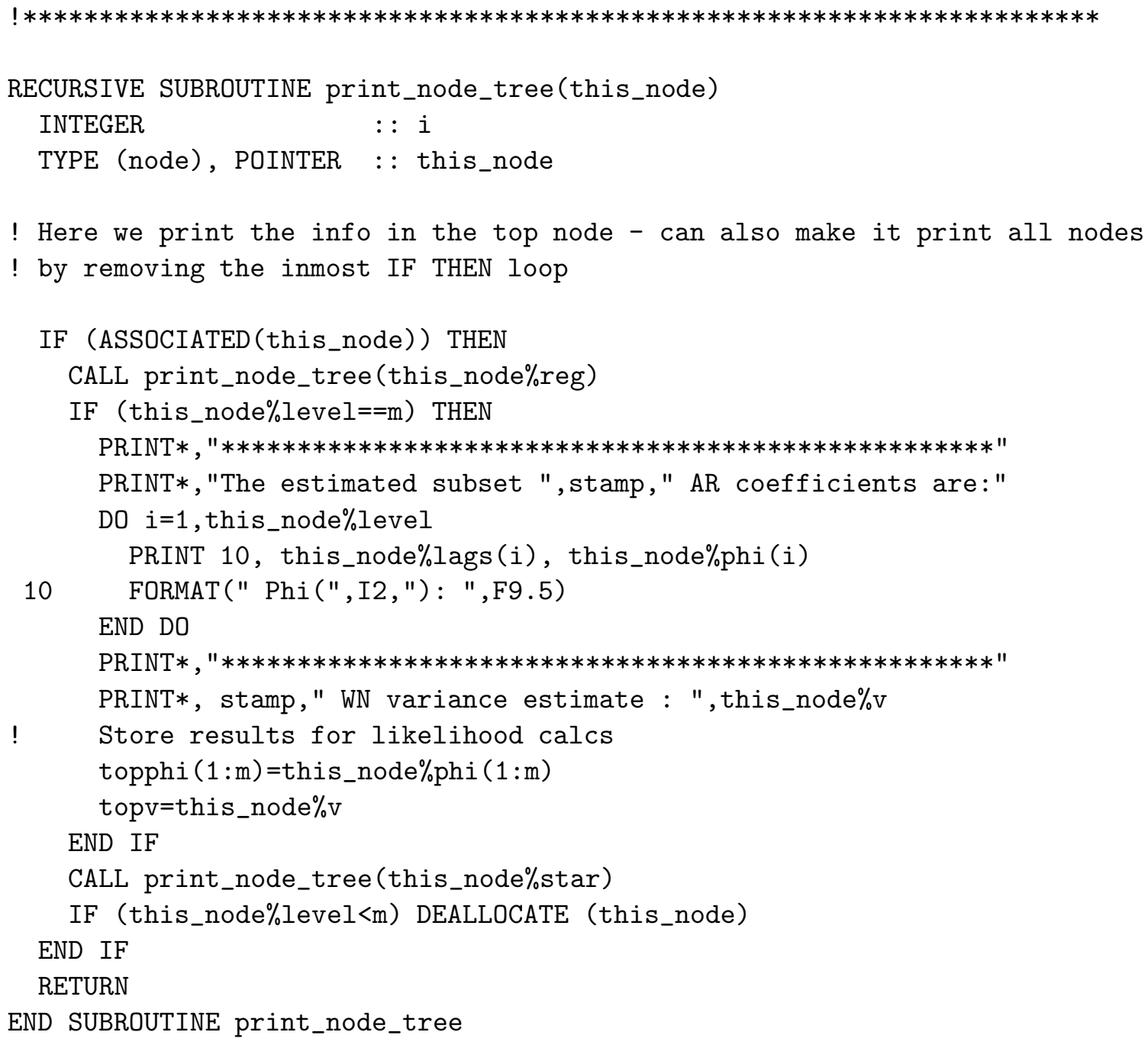




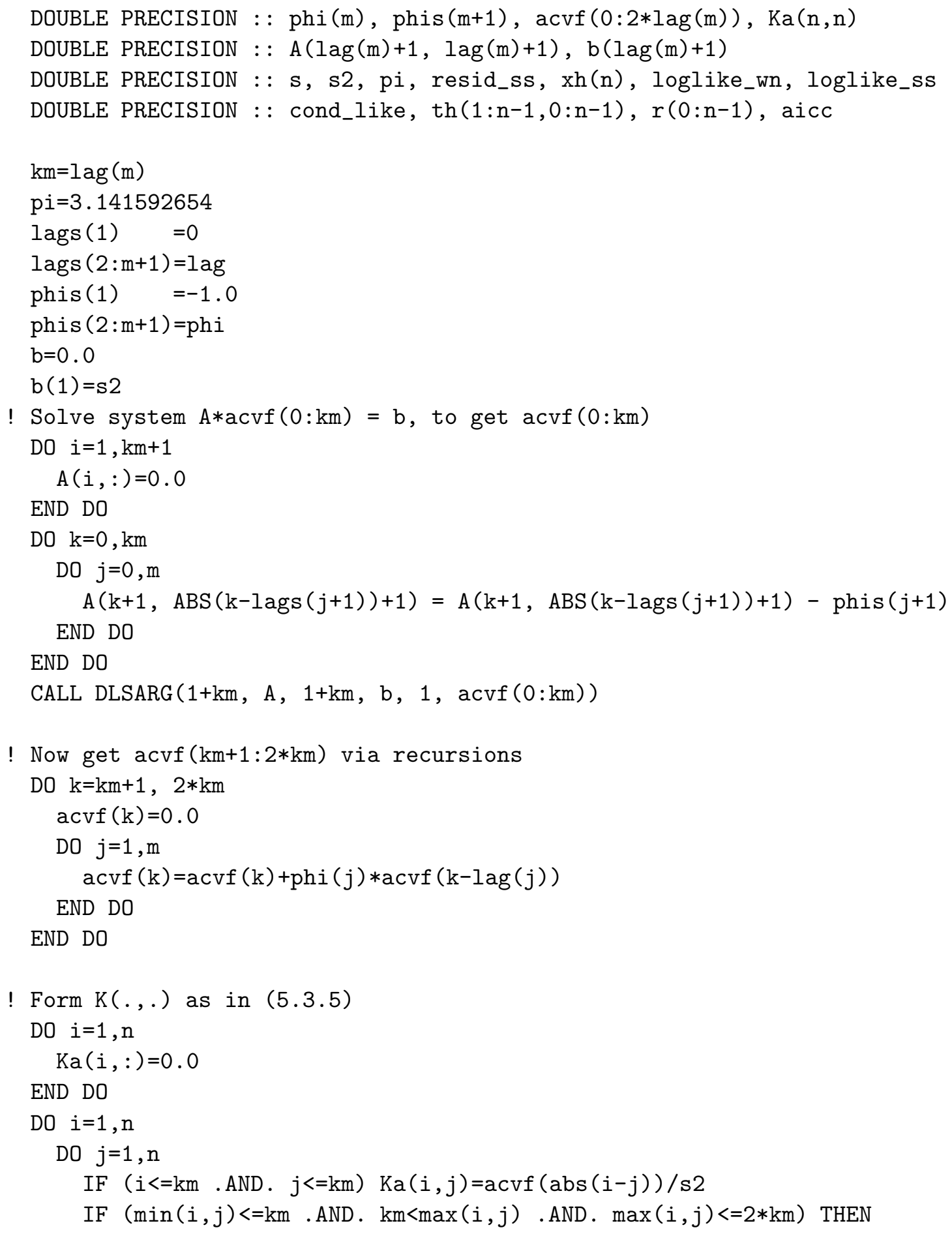




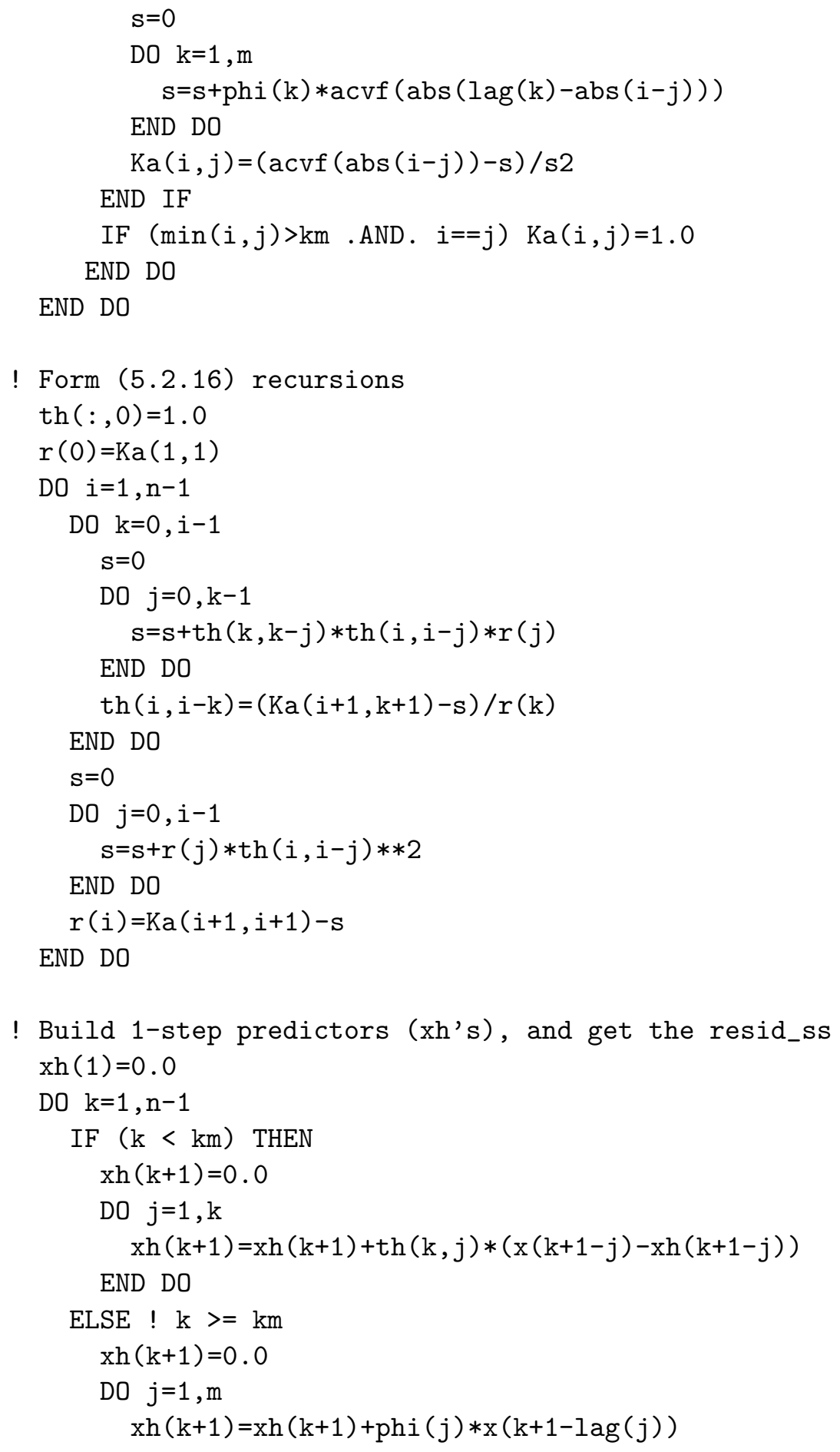

! Build 1-step predictors (xh's), and get the resid_ss $\operatorname{xh}(1)=0.0$ 


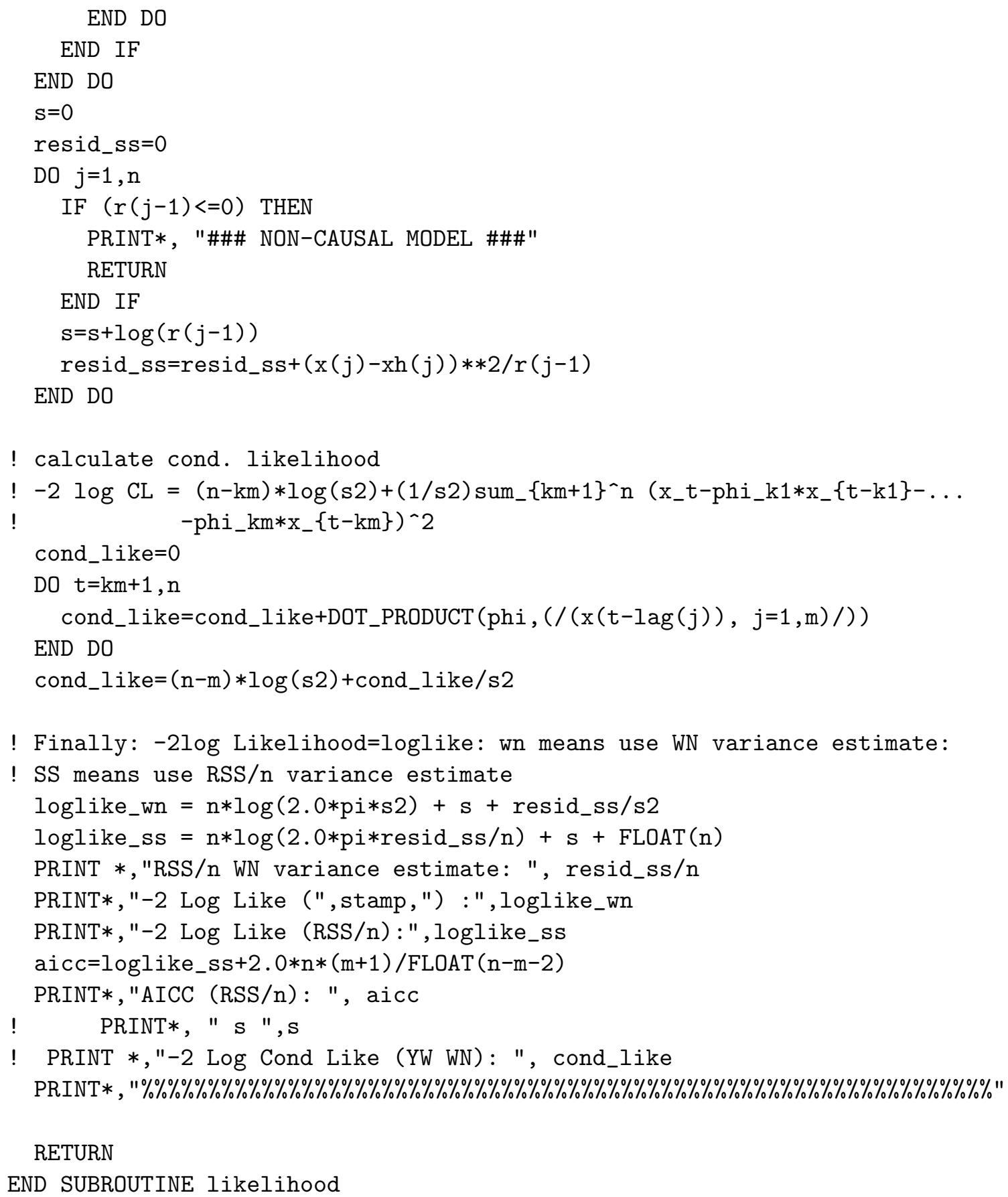




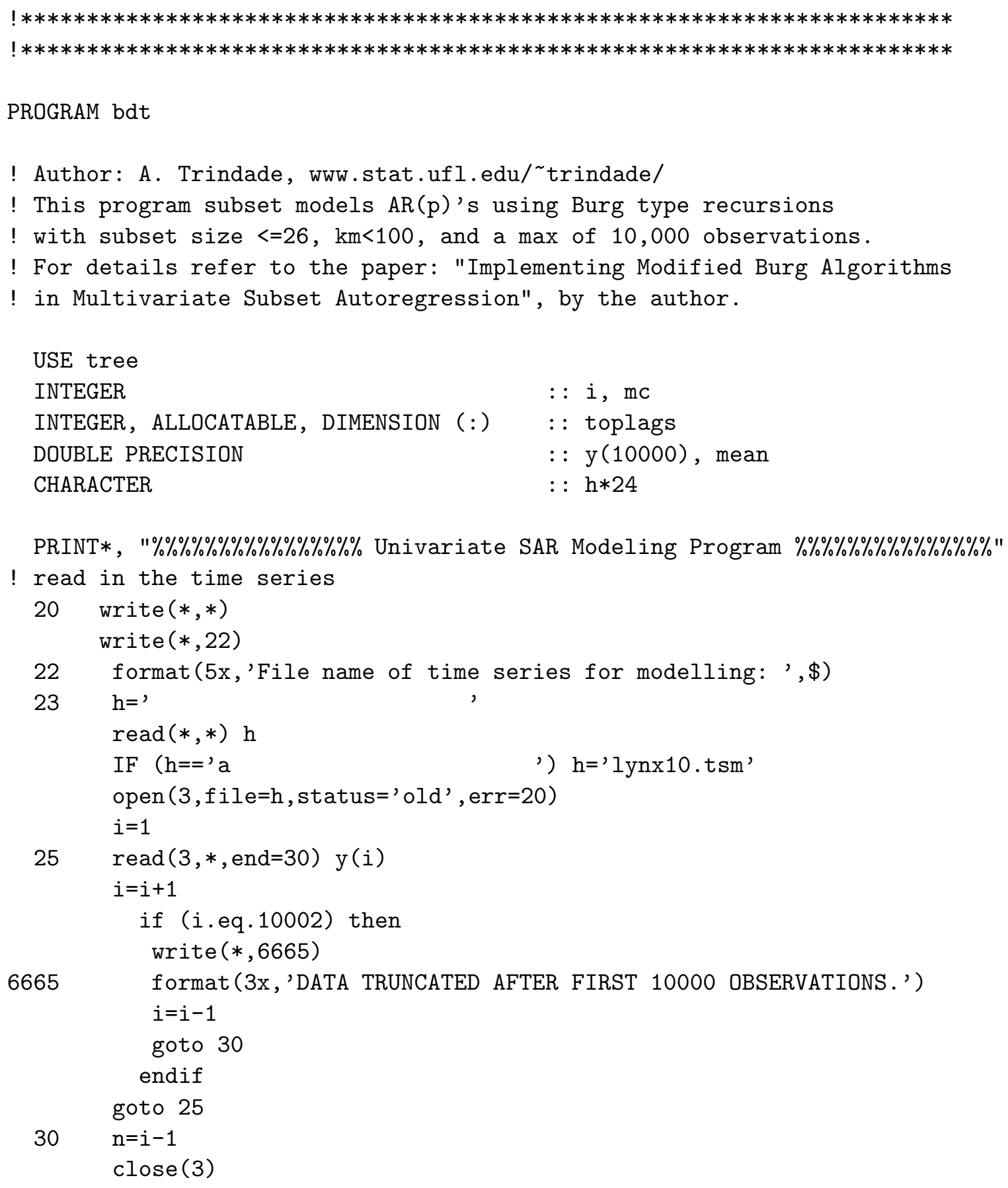




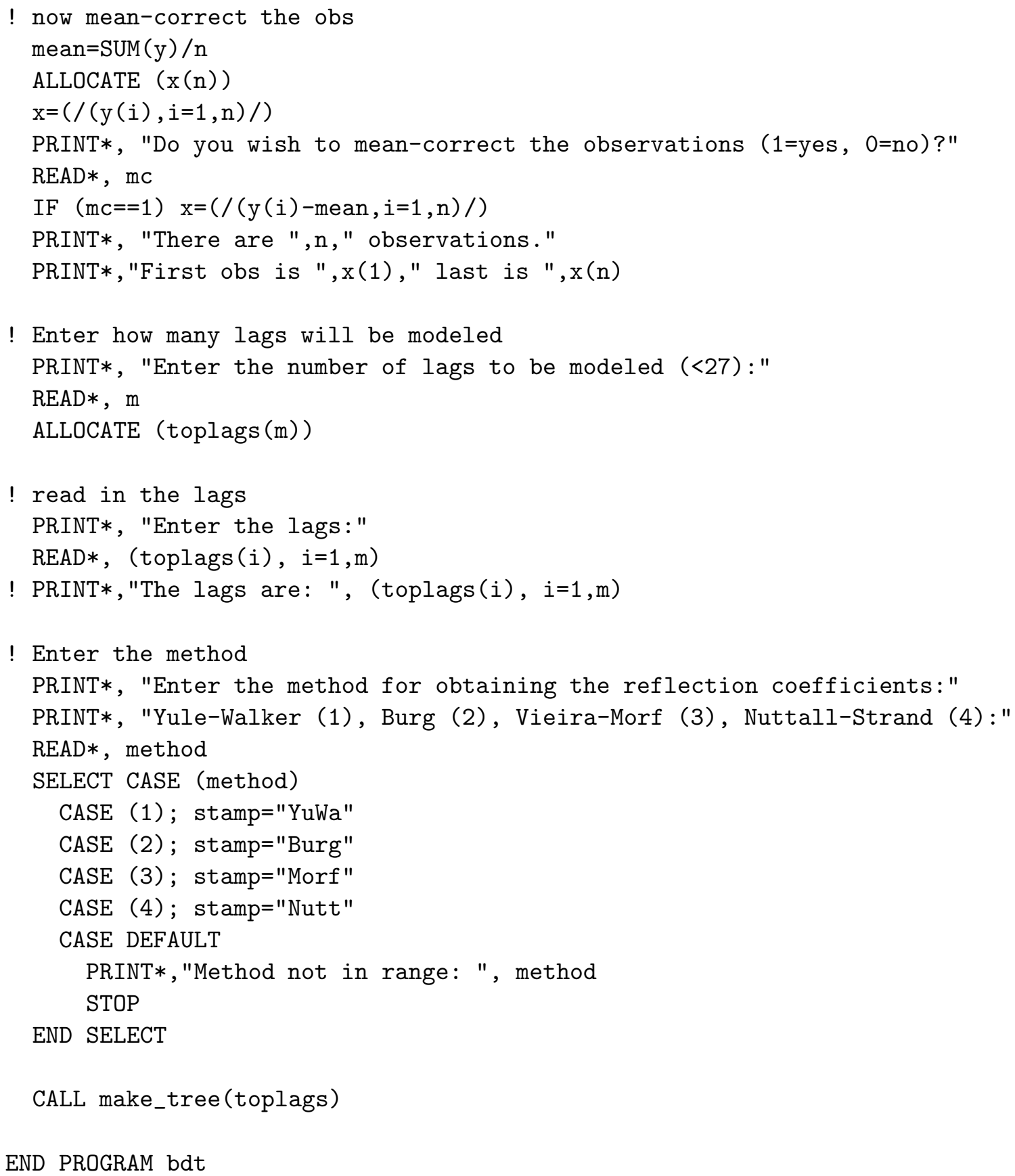




\section{C.2 Code for BDT2.F90}

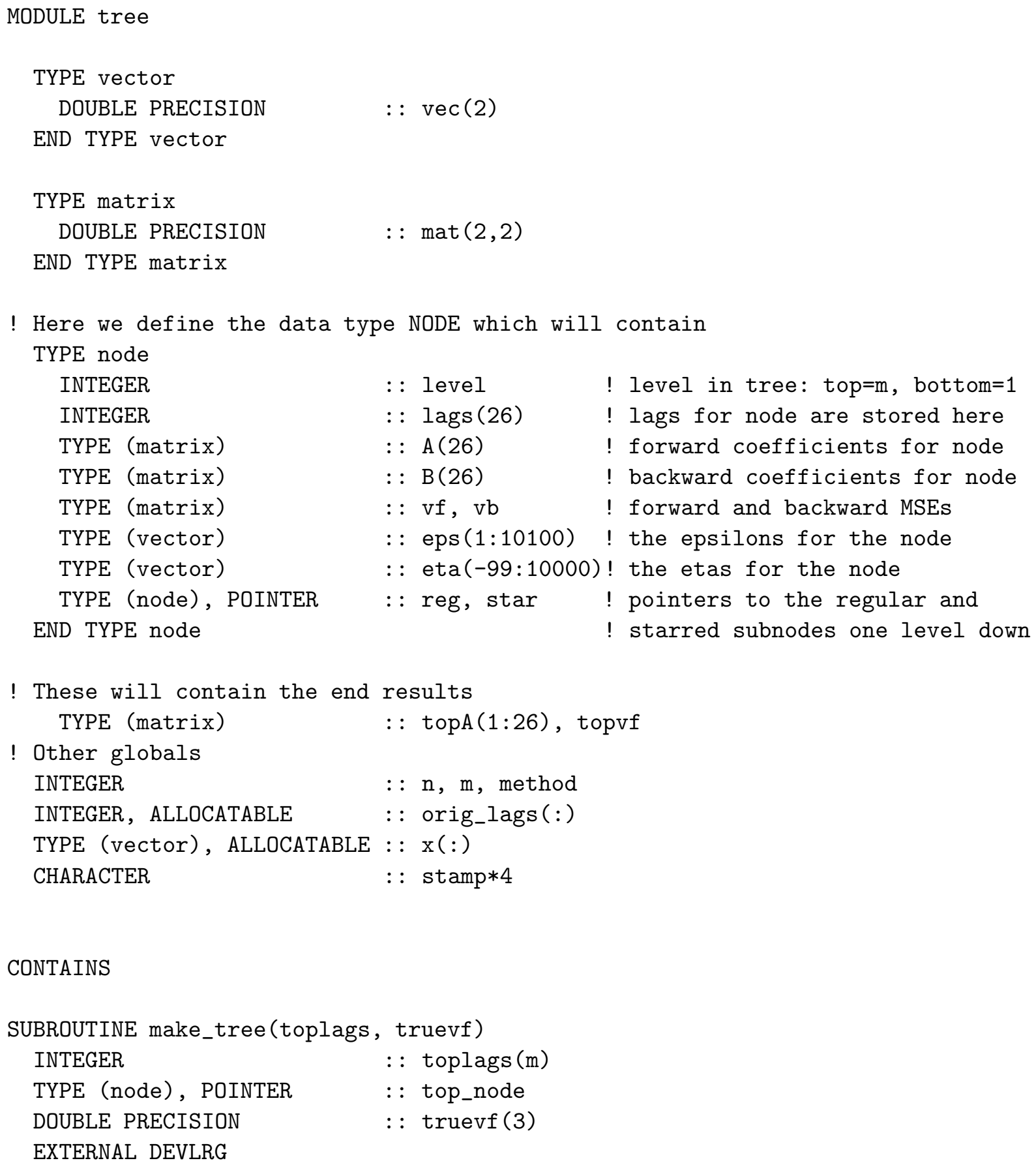

\section{CONTAINS}

SUBROUTINE make_tree(toplags, truevf)

EXTERNAL DEVLRG 


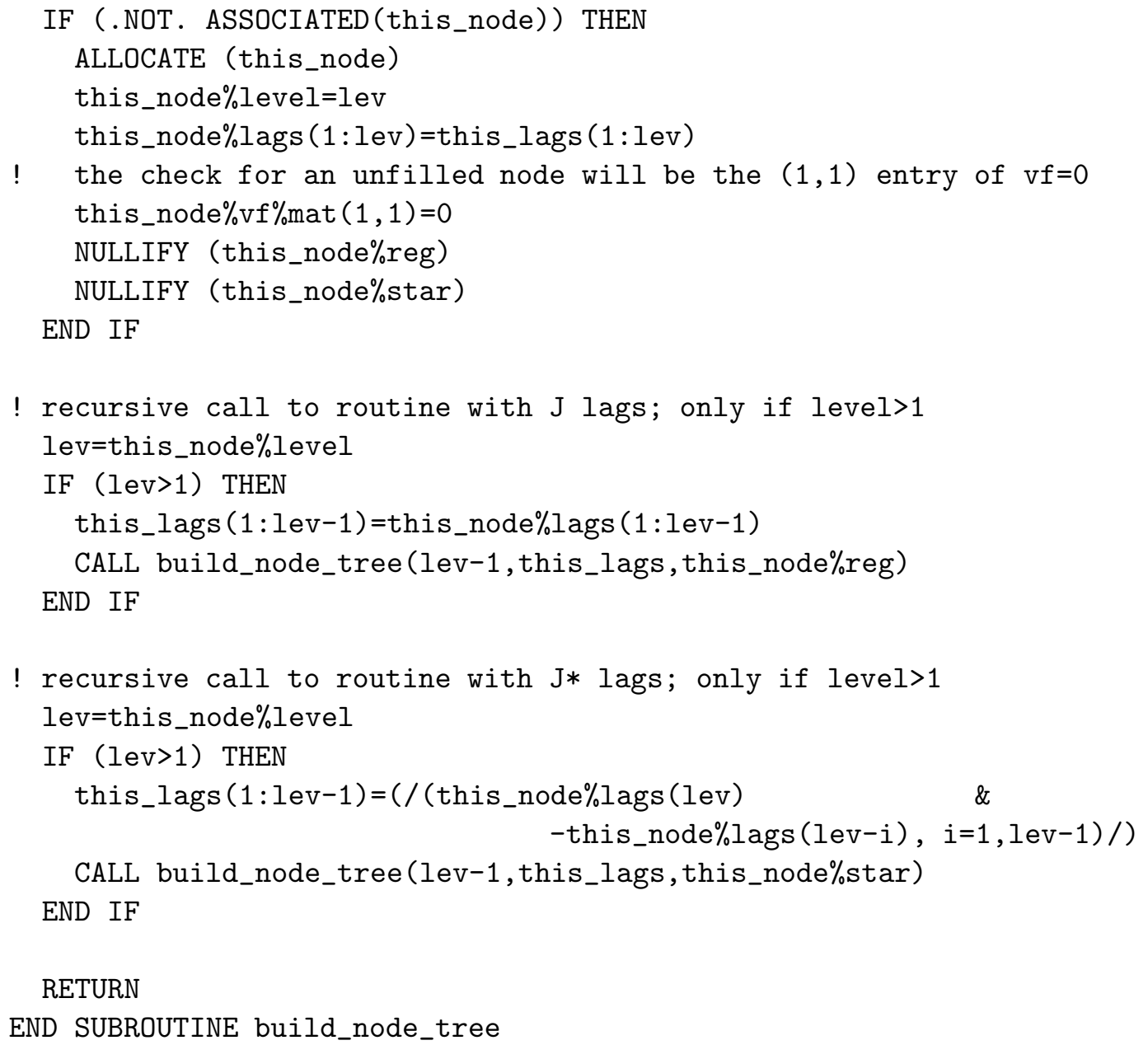




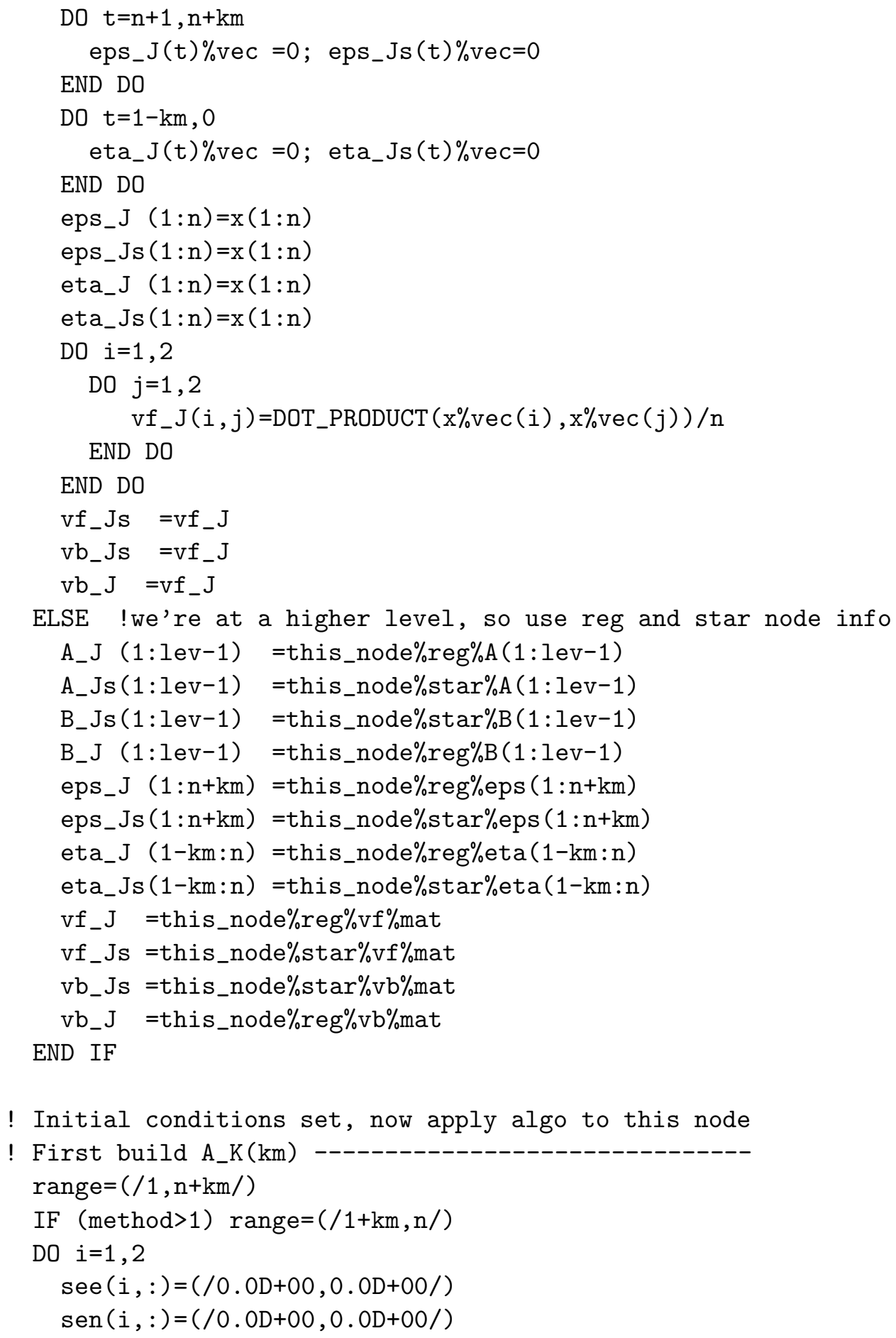

! Initial conditions set, now apply algo to this node

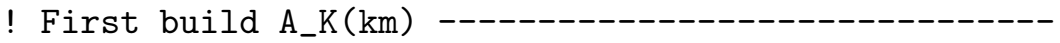
range $=(/ 1, \mathrm{n}+\mathrm{km} /)$

IF (method $>1$ ) range $=(/ 1+k m, n /$ )

DO $i=1,2$

$\operatorname{see}(i,:)=(/ 0.0 D+00,0.0 D+00 /)$

$\operatorname{sen}(i,:)=(/ 0.0 \mathrm{D}+00,0.0 \mathrm{D}+00 /)$ 
$\operatorname{snn}(i,:)=(/ 0.0 \mathrm{D}+00,0.0 \mathrm{D}+00 /)$

END DO

DO $t=$ range (1), range (2)

DO $i=1,2$

DO $j=1,2$

$\operatorname{see}(i, j)=\operatorname{see}(i, j)+e p s_{-} J(t) \% \operatorname{vec}(i) * \operatorname{eps} s_{-} J(t) \% \operatorname{vec}(j)$

$\operatorname{sen}(i, j)=\operatorname{sen}(i, j)+e p s_{-} J(t) \% \operatorname{vec}(i) * e t a_{-} J s(t-k m) \% \operatorname{vec}(j)$

$\operatorname{snn}(i, j)=\operatorname{snn}(i, j)+e t a_{-} J s(t-k m) \% \operatorname{vec}(i) * e t a_{-} J s(t-k m) \% \operatorname{vec}(j)$

END DO

END DO

END DO

! form I

$\operatorname{Id}(1,:)=(/ 1.0 \mathrm{D}+00,0.0 \mathrm{D}+00 /)$

$\operatorname{Id}(2,:)=(/ 0.0 \mathrm{D}+00,1.0 \mathrm{D}+00 /)$

! get the inverse of $v f_{-} J$, put into vfinv

CALL DLINRG (2,vf_J,2,vfinv,2)

SELECT CASE (method)

CASE (1)

! get the inverse of $\mathrm{vb}_{-} \mathrm{Js}$, put into vbsinv

CALL DLINRG (2,vb_Js, 2, vbsinv, 2)

! finally, get $A_{-} K(\mathrm{~km})$ \& $B_{-} K s(\mathrm{~km})$

$A_{-} \mathrm{K}=$ MATMUL (sen, vbsinv)/FLOAT (n)

B_Ks=MATMUL (TRANSPOSE (sen), vfinv)/FLOAT (n)

CASE (2)

$\mathrm{B}=$ sen+MATMUL (MATMUL (vfinv, sen), vb_Js)

CALL KRON $(2$, snn, Id, term1_left)

CALL KRON (2, MATMUL (vb_Js, vb_Js), MATMUL (MATMUL (vfinv, see), vfinv), \& \& term1_right)

term1=term1_left+term1_right

CALL DLINRG (4, term1,4,A,4)

! finally, build elements of $A_{-} K$ piecemeal

DO $i=1,2$

$A_{-} K(i, 1)=A(i, 1) * B(1,1)+A(i, 2) * B(2,1)+A(i, 3) * B(1,2)+A(i, 4) * B(2,2)$

END DO

DO $i=3,4$

$A_{-} K(i-2,2)=A(i, 1) * B(1,1)+A(i, 2) * B(2,1)+A(i, 3) * B(1,2)+A(i, 4) * B(2,2)$

END DO

! Finished building $A_{-} K$--------- now get $B_{-} K s$ from it

B_Ks $=$ MATMUL (MATMUL (vb_Js, TRANSPOSE (A_K)), vfinv)

CASE (3) 


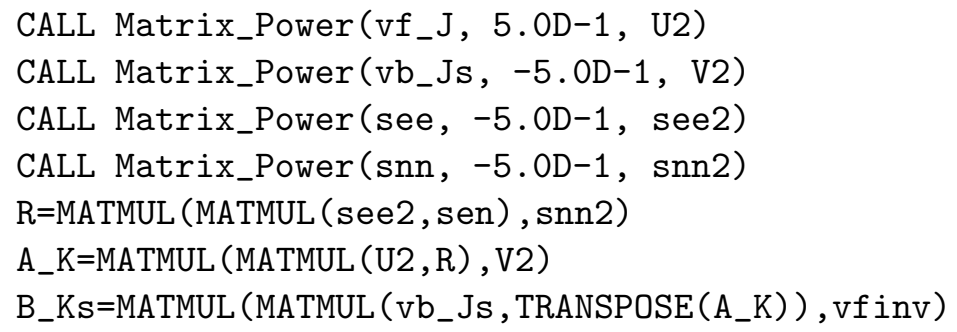




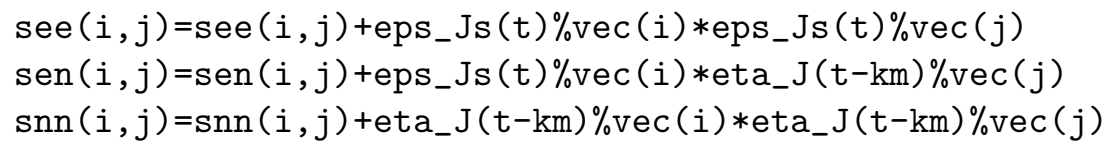




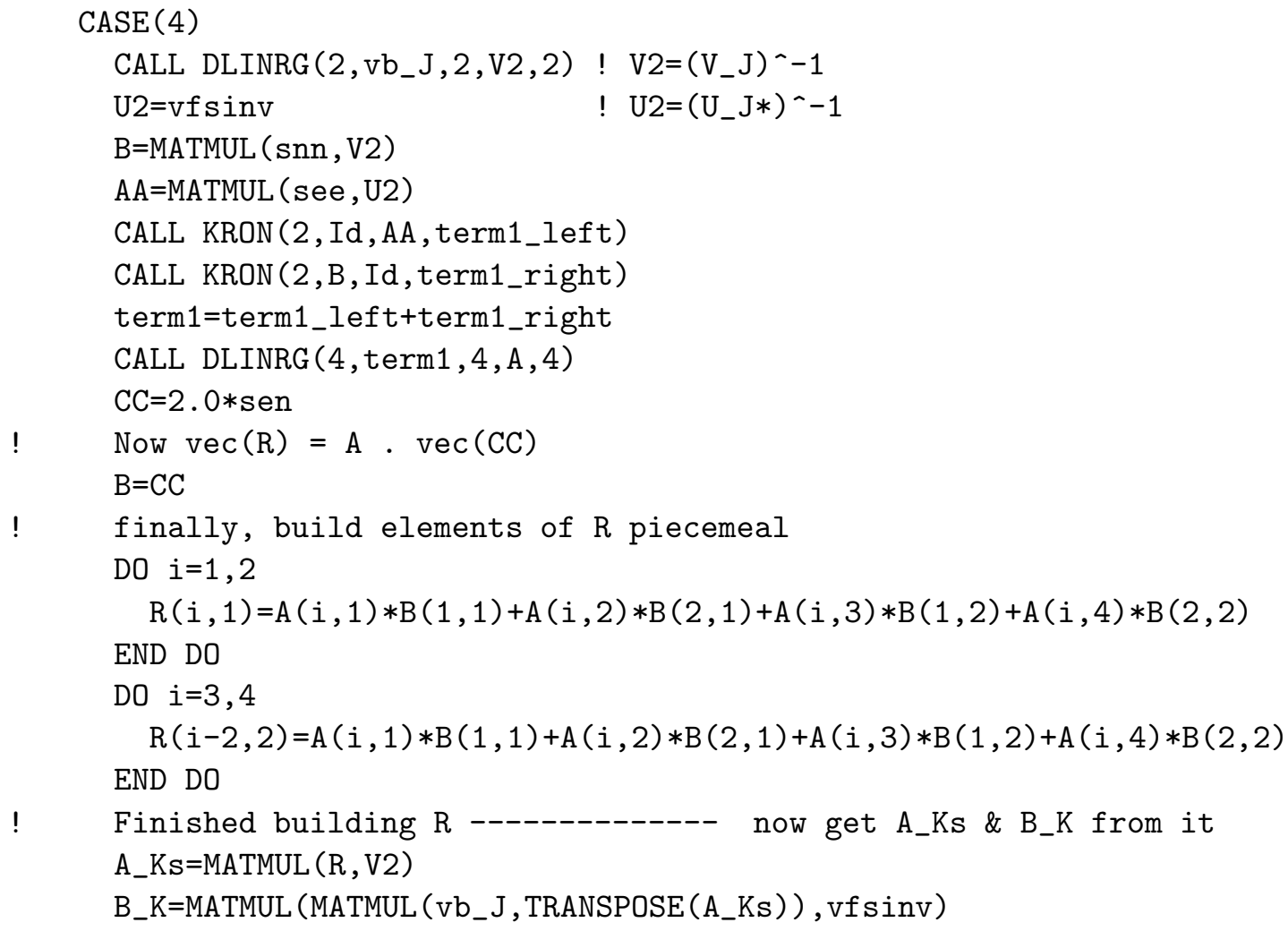




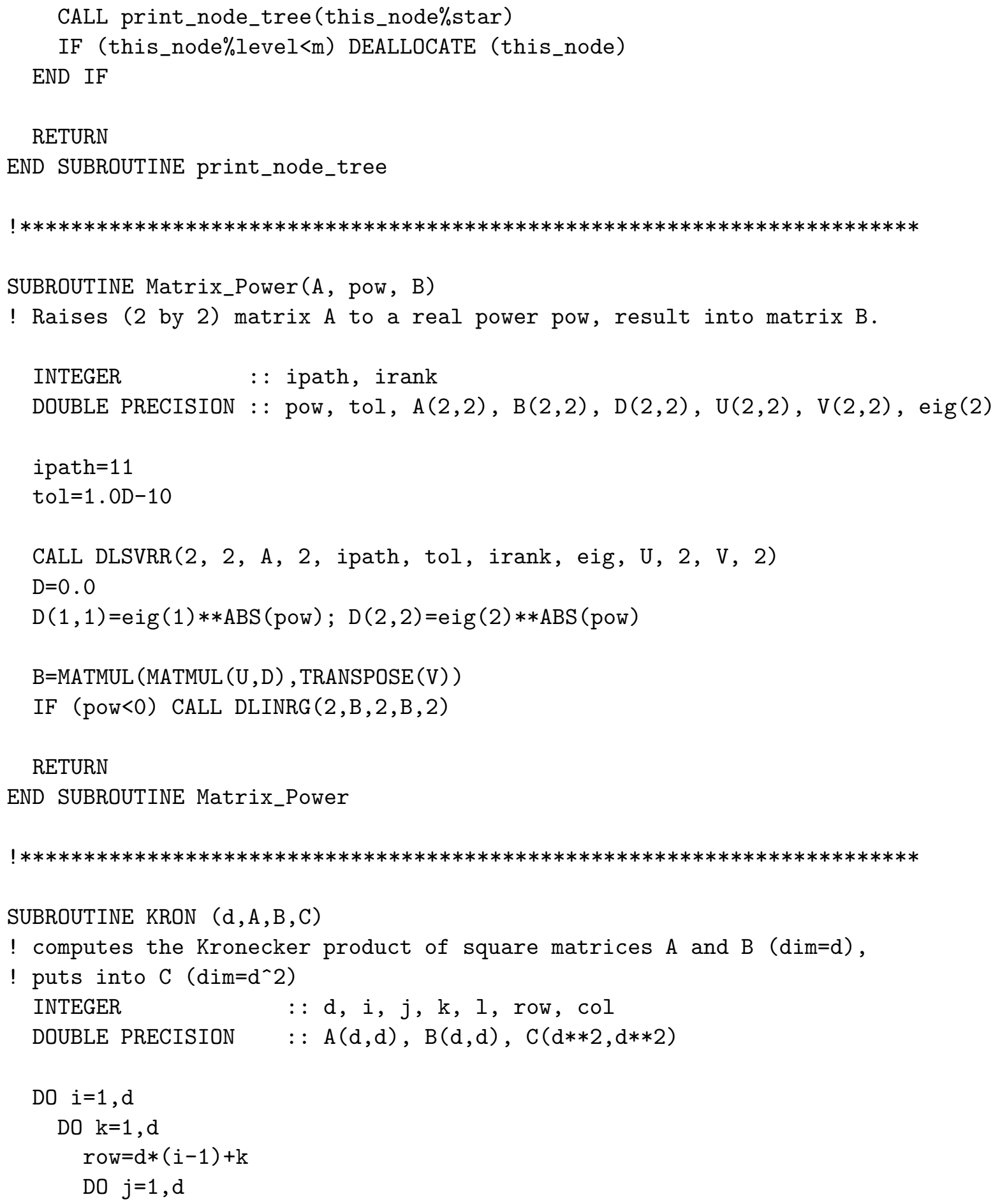




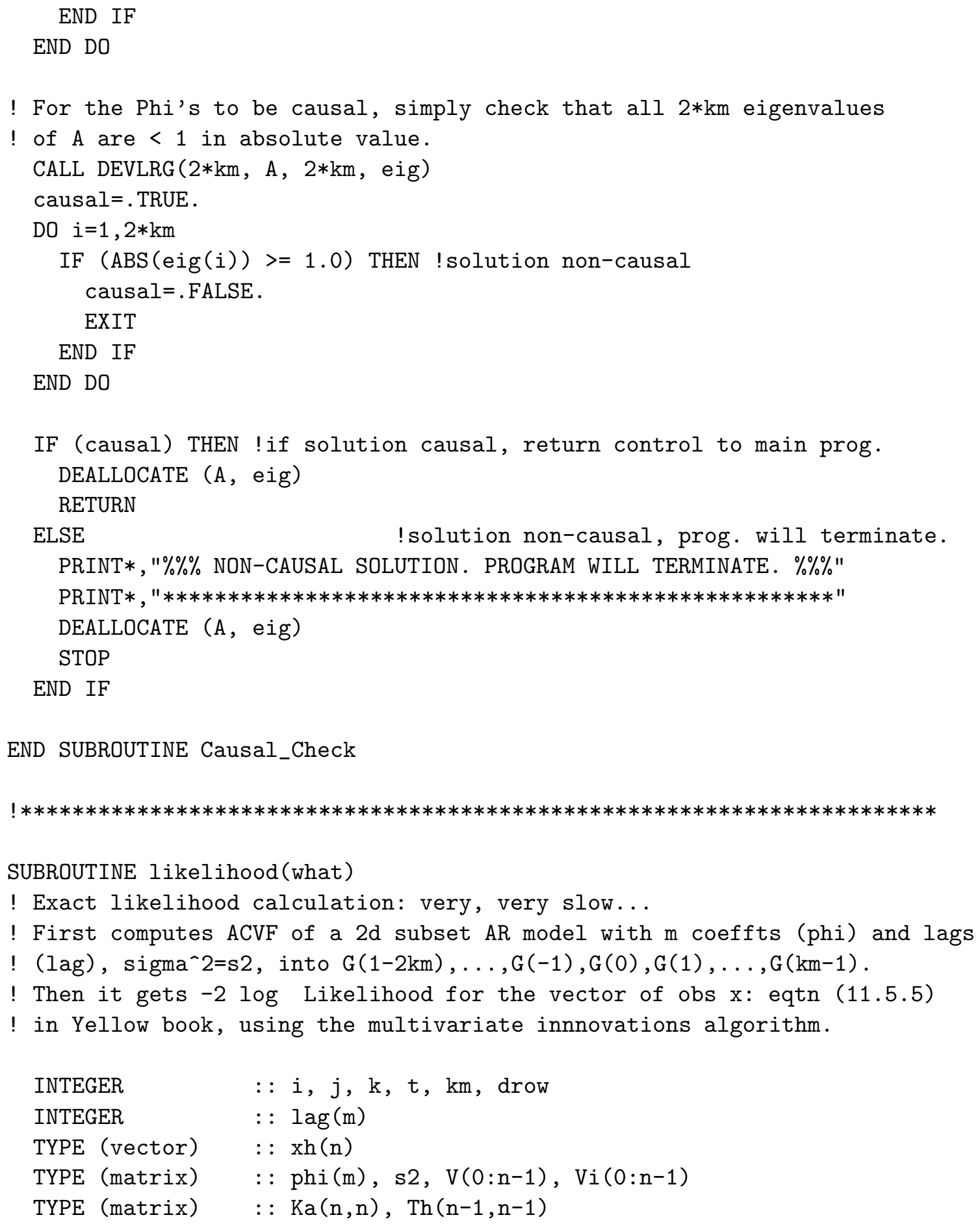




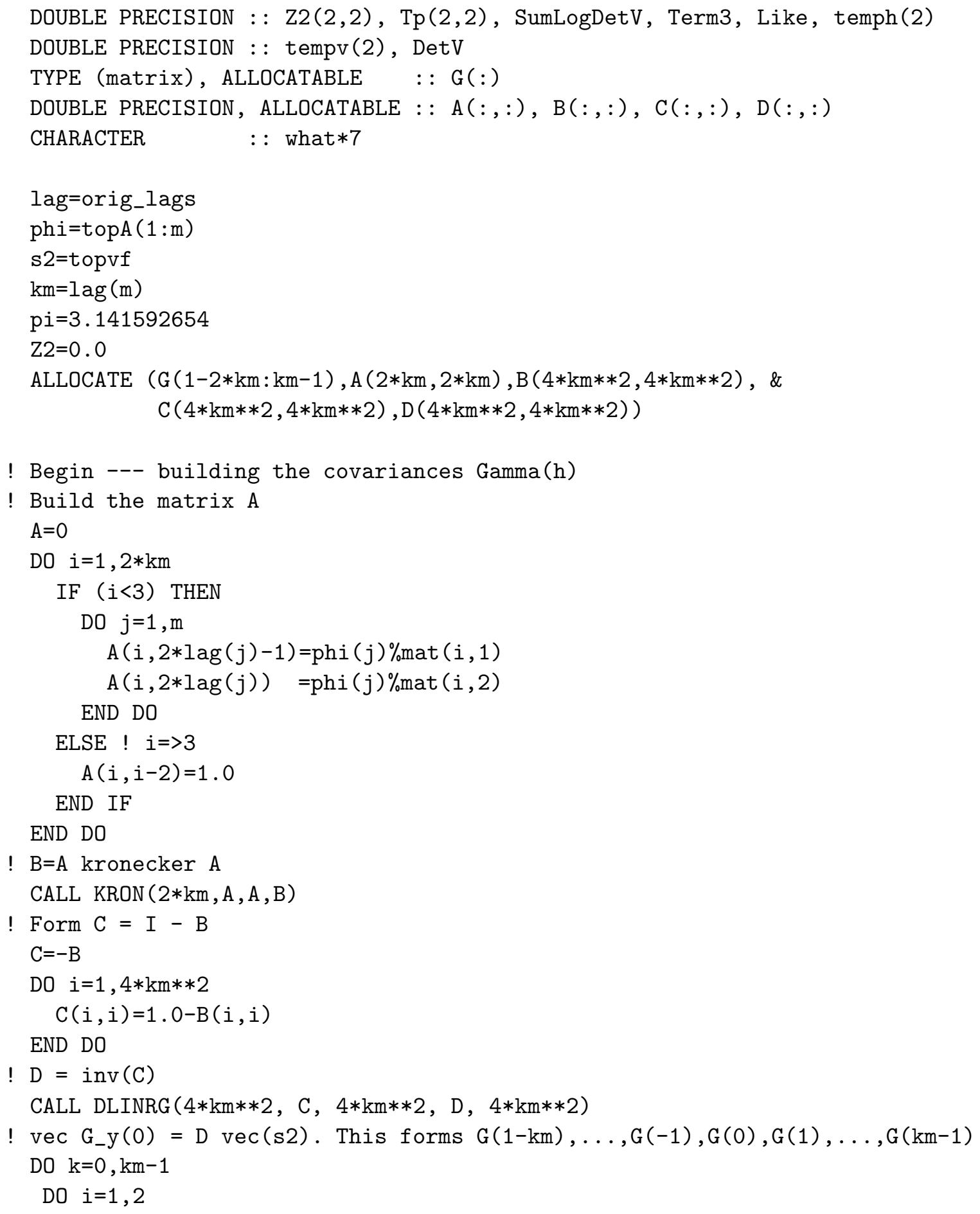




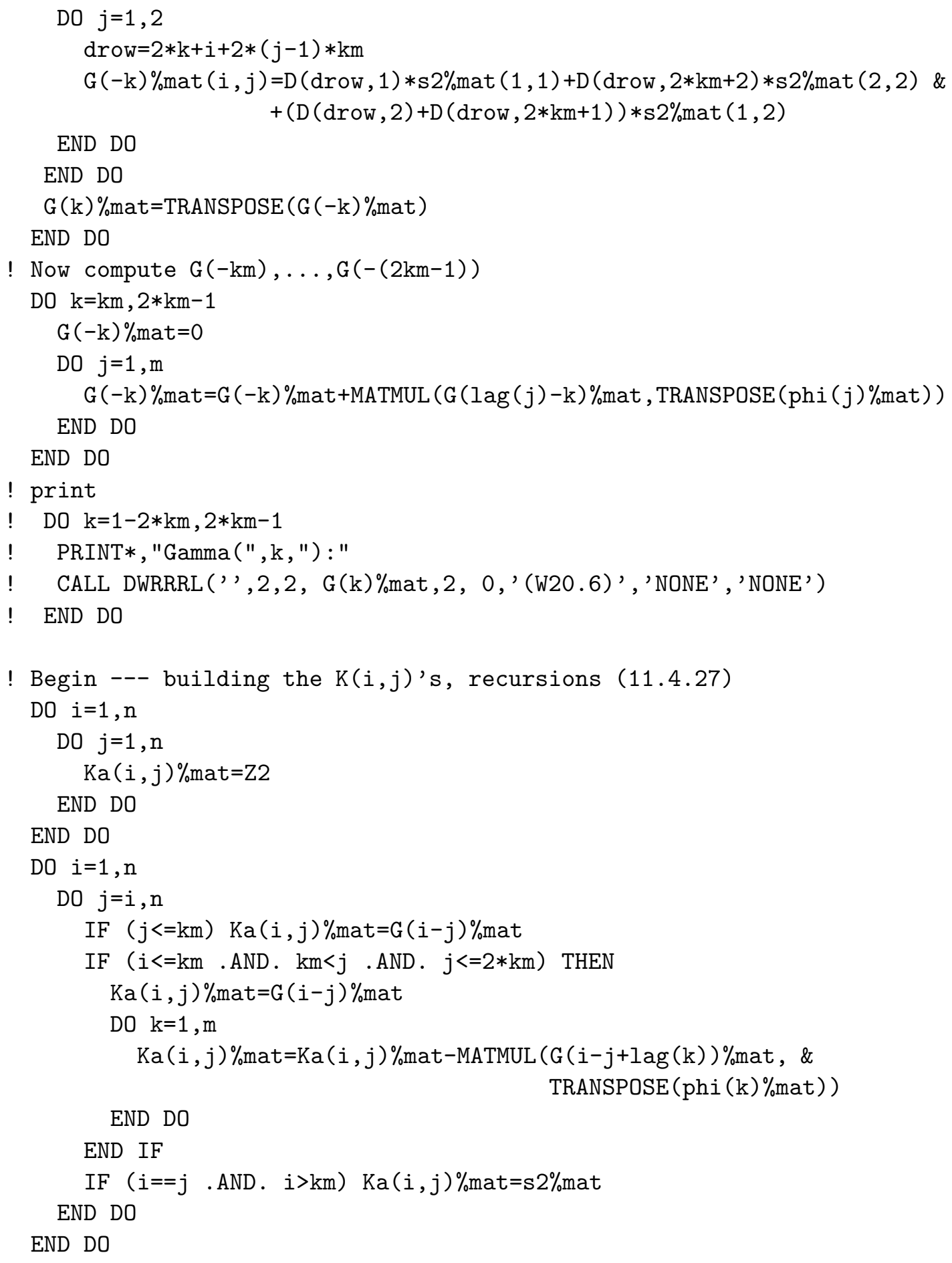




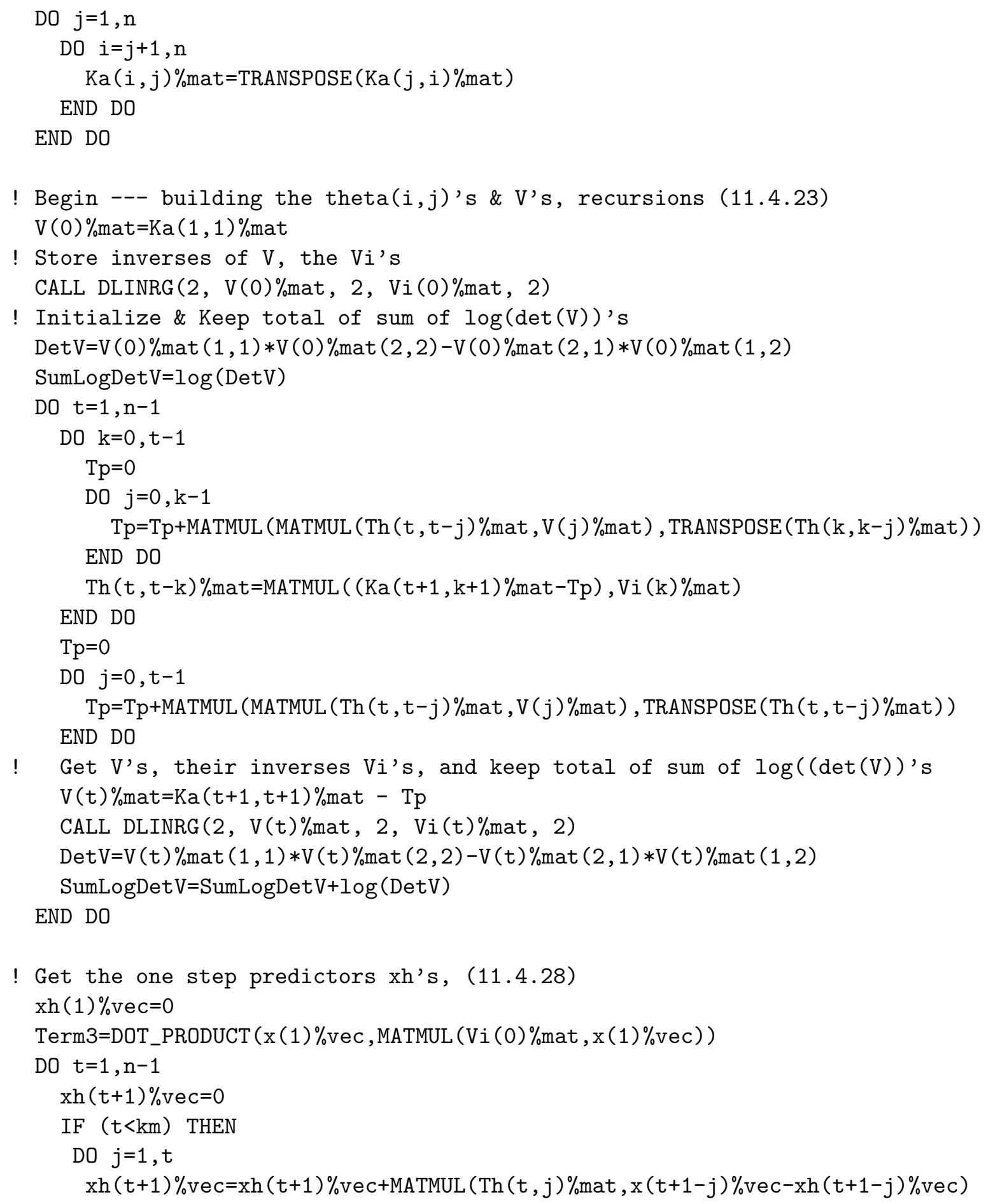

! Get the one step predictors xh's, (11.4.28)

$\operatorname{xh}(1) \%$ vec $=0$ 


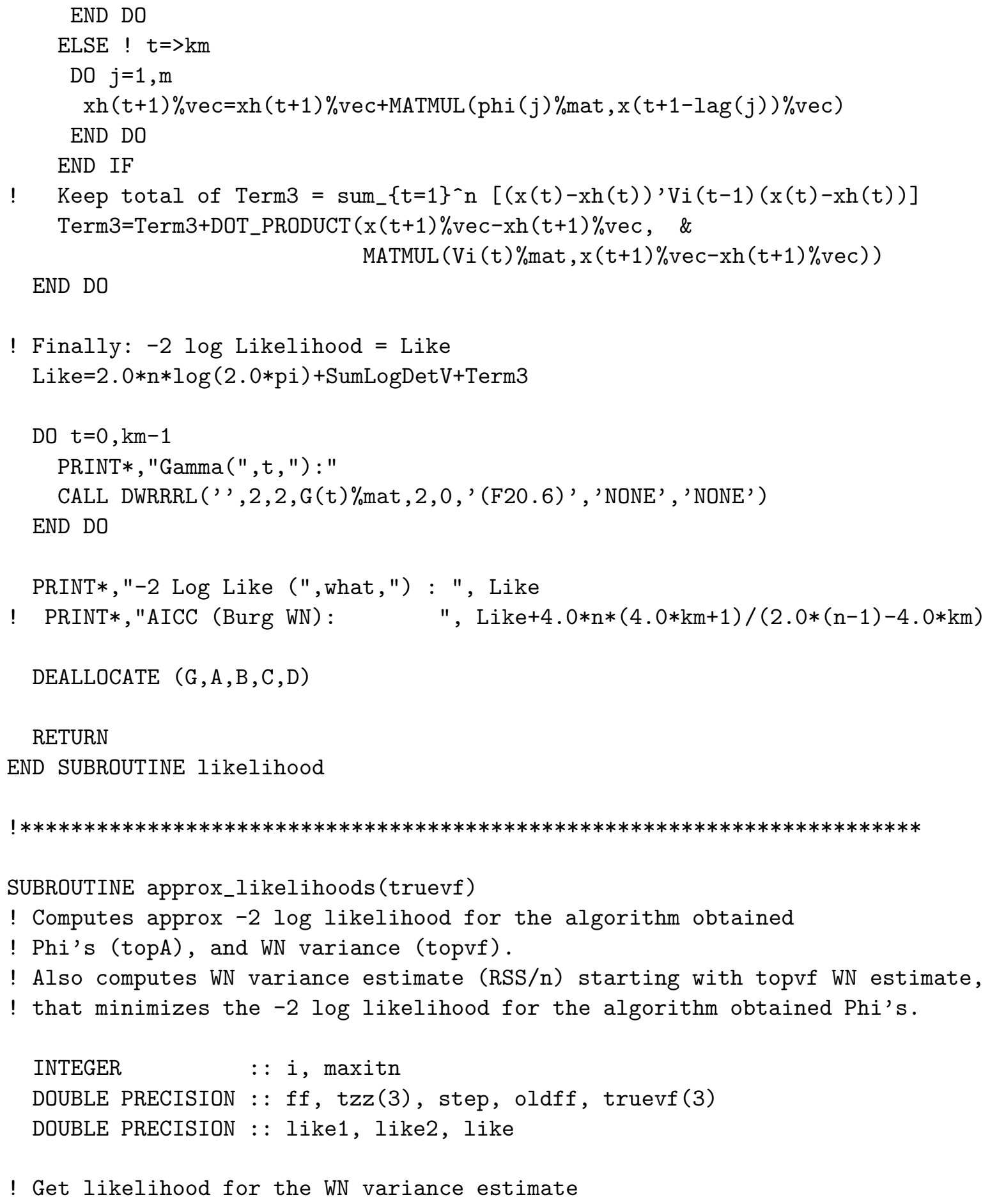




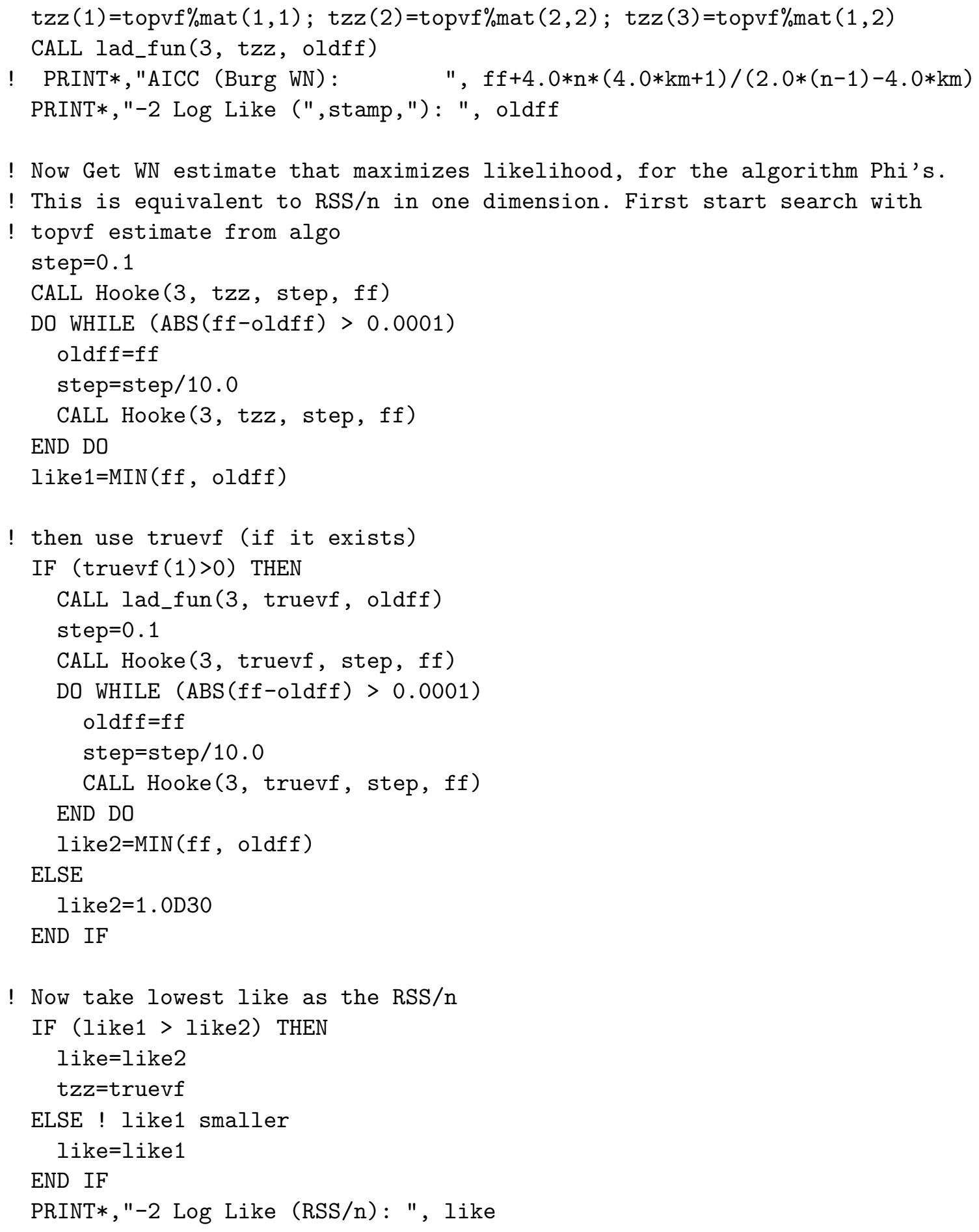


! get the RSS/n matrix

topvf $\%$ mat $(1,1)=\operatorname{tzz}(1) ; \operatorname{topvf} \% \operatorname{mat}(2,2)=\operatorname{tzz}(2)$

topvf $\% \operatorname{mat}(1,2)=\operatorname{tzz}(3) ; \operatorname{topvf} \% \operatorname{mat}(2,1)=\operatorname{tzz}(3)$

PRINT*, " "

PRINT*,"Estimated RSS/n (forward) WN covariance matrix:"

CALL DWRRRL (', , 2, 2, topvf\%mat, 2,0,' (F20.6) ', 'NONE' , 'NONE')

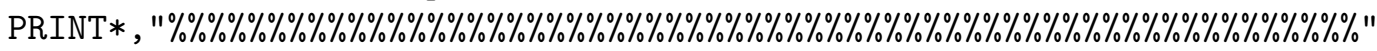

RETURN

END SUBROUTINE approx_likelihoods

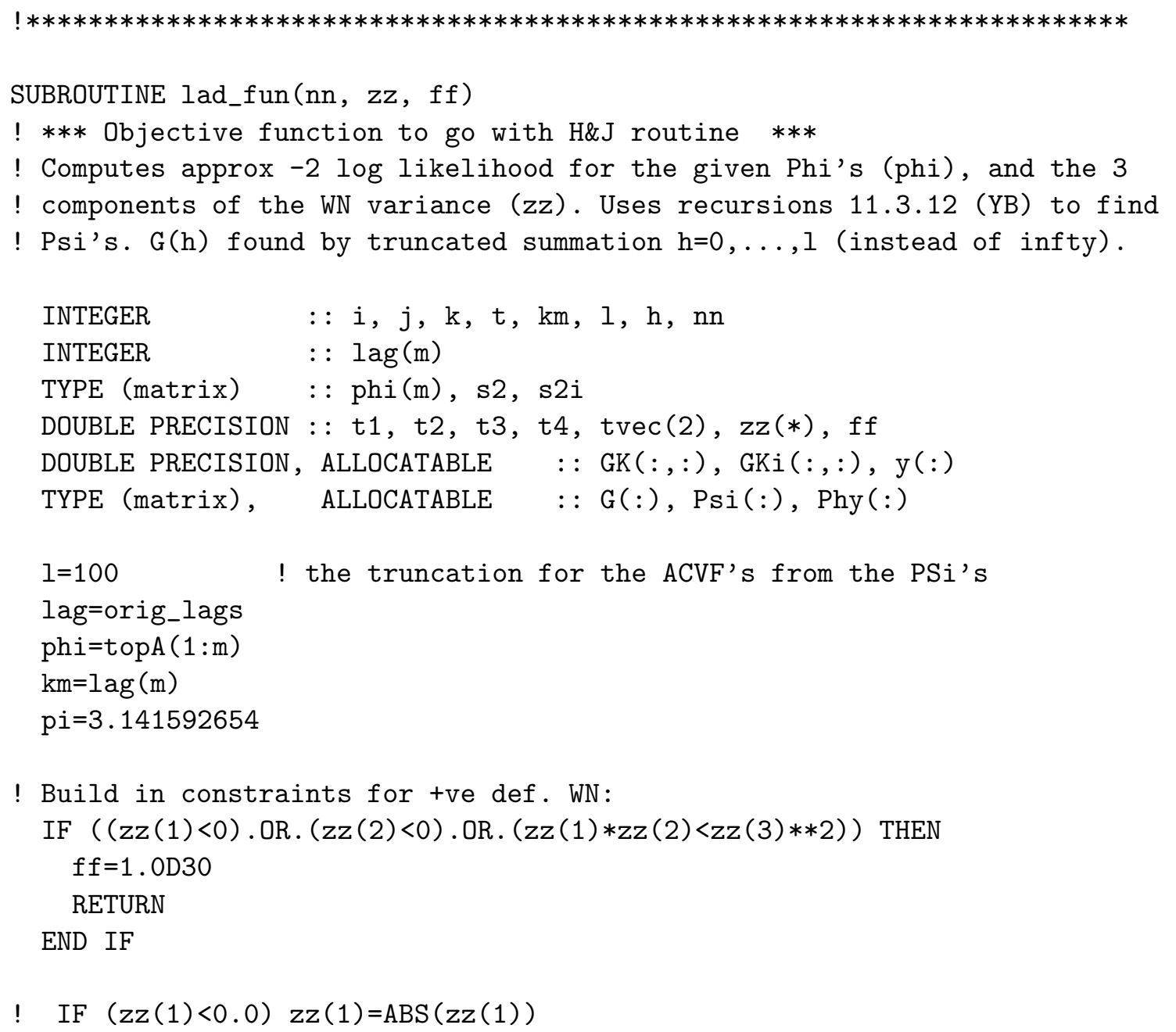




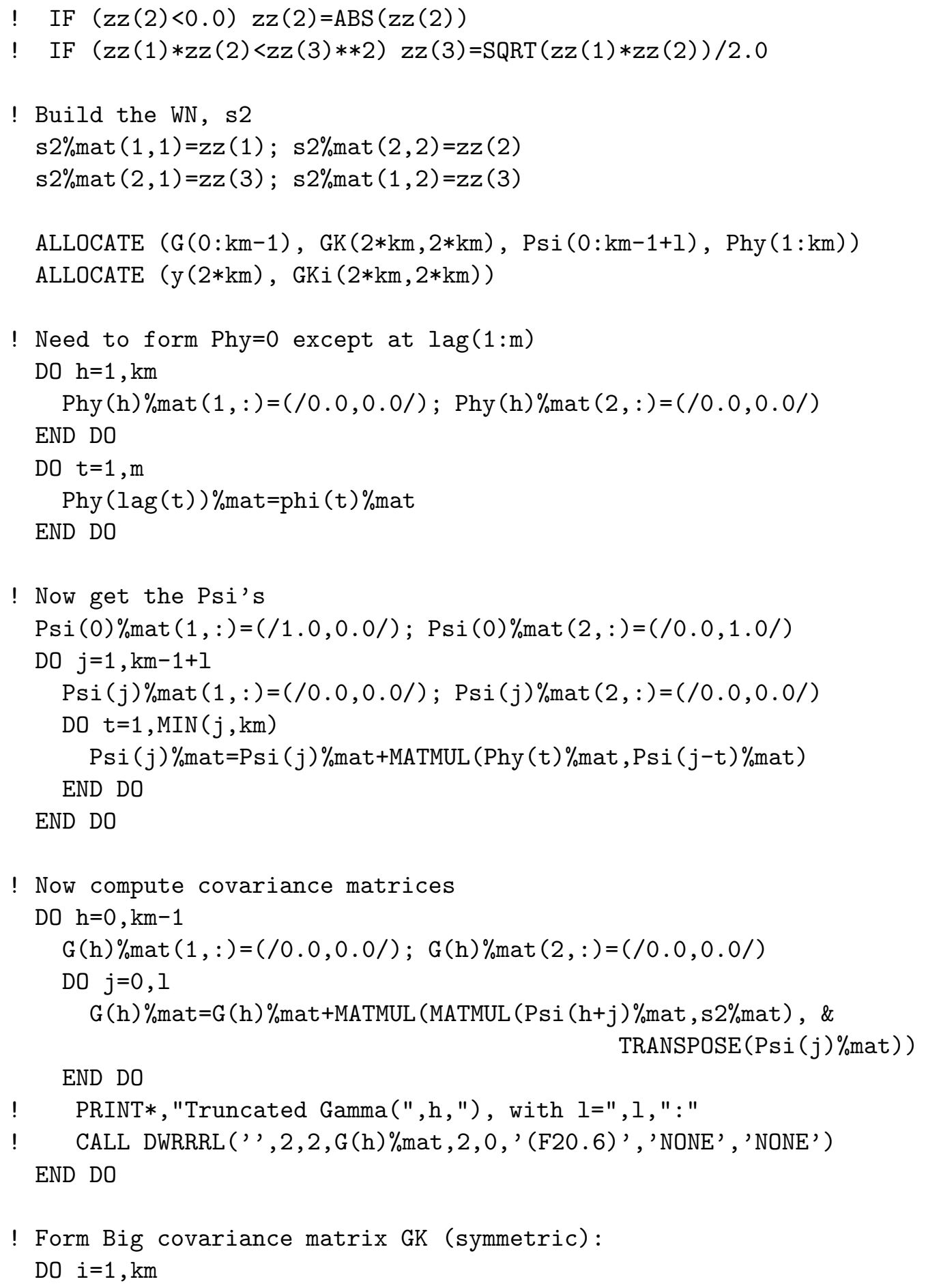

! Now compute covariance matrices

! Form Big covariance matrix GK (symmetric): DO $i=1, k m$ 


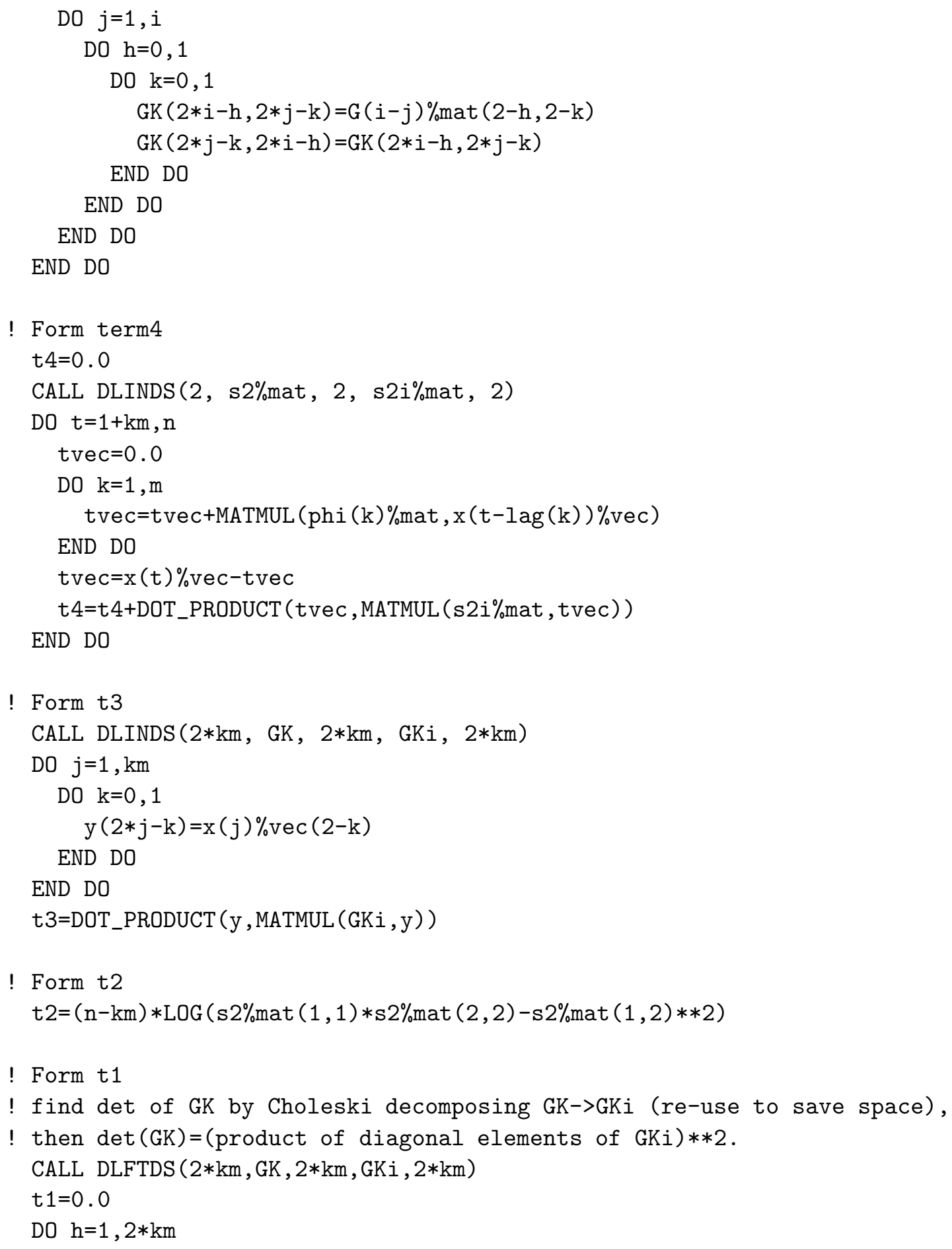




\begin{tabular}{|c|c|}
\hline & goto 3600 \\
\hline ! & \\
\hline ! & Exploratory moves \\
\hline$!$ & \\
\hline 3100 & do $3105 j=1, m$ \\
\hline 3105 & $x 2(j)=x 1(j)$ \\
\hline & do $3160 i=1, m$ \\
\hline & do $3115 j=1, m$ \\
\hline 3115 & $c(j)=x 2(j)$ \\
\hline & $c(i)=x 1(i)+e r$ \\
\hline & call lad_fun (p,c,fv) \\
\hline 3120 & if (fv.lt.min) then \\
\hline & $\min =f \mathrm{v}$ \\
\hline & $x 2(i)=c(i)$ \\
\hline & endif \\
\hline & $c(i)=x 1(i)-e r$ \\
\hline & call lad_fun $(p, c, f v)$ \\
\hline 3130 & if (fv.lt.min) then \\
\hline & $\min =f v$ \\
\hline & $\mathrm{x} 2(i)=\mathrm{c}(i)$ \\
\hline & endif \\
\hline 3160 & continue \\
\hline & $f l g=1$ \\
\hline & do $3180 \quad i=1, m$ \\
\hline & if $(x 1(i) \cdot n e \cdot x 2(i)) f l g=0$ \\
\hline 3180 & continue \\
\hline & $\begin{array}{c}\text { if (flg.eq.0) goto } 3195 \\
\text { er }=\text { er } / 2\end{array}$ \\
\hline & $\operatorname{if}(e r .1 t . a c) f i=1$ \\
\hline & if (ik.eq.0) goto 3285 \\
\hline & if (ik.eq.1) goto 3605 \\
\hline & goto 3100 \\
\hline 3195 & if (ik.eq.0) goto 3285 \\
\hline & if (ik.eq.1) goto 3605 \\
\hline$!$ & \\
\hline ! & Pattern moves \\
\hline$!$ & \\
\hline 3200 & do $3230 i=1, m$ \\
\hline 3230 & $\mathrm{x} 1(i)=2 * x 2(i)-x 1(i)$ \\
\hline & do $3260 i=1, m$ \\
\hline
\end{tabular}




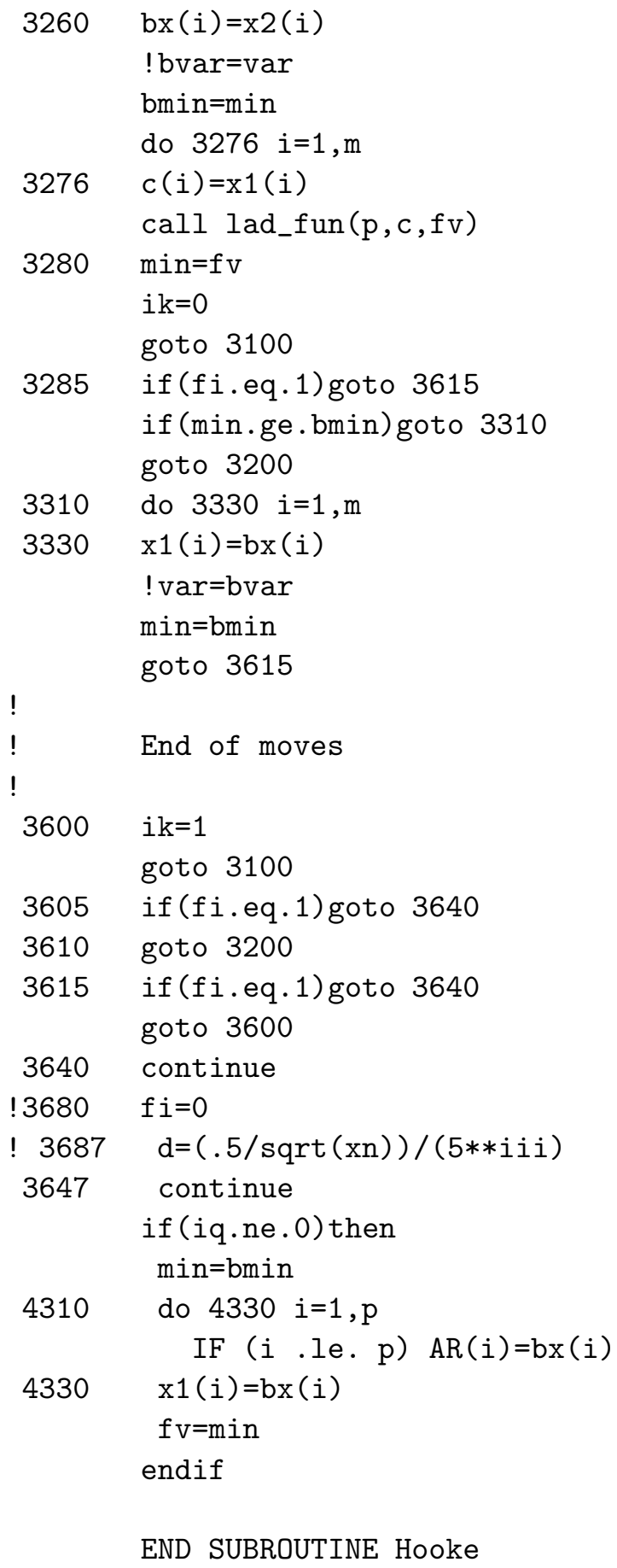


! this program subset models AR(p)'s for 2-dimensional time series, using

! Burg type recursions, with subset size $<=26, \mathrm{~km}<100$, and a max of 10000 obs .

USE tree

INTEGER

INTEGER, ALLOCATABLE, DIMENSION (:)

TYPE (vector)

CHARACTER

DOUBLE PRECISION

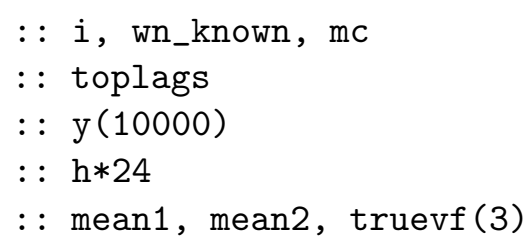

PRINT*, " $\% \% \% \% \% \% \% \% \% \% \% \% \%$ Bivariate SVAR Modeling Program \%\%\%\%\%\%\%\%\%\%\%\% $\% \% \%$ "

! read in the time series from a data file

20 write $(*, *)$ write $(*, 22)$

22 format( $5 \mathrm{x}$, 'Enter file name of time series for modelling: ',\$)

$23 \mathrm{~h}=$ '

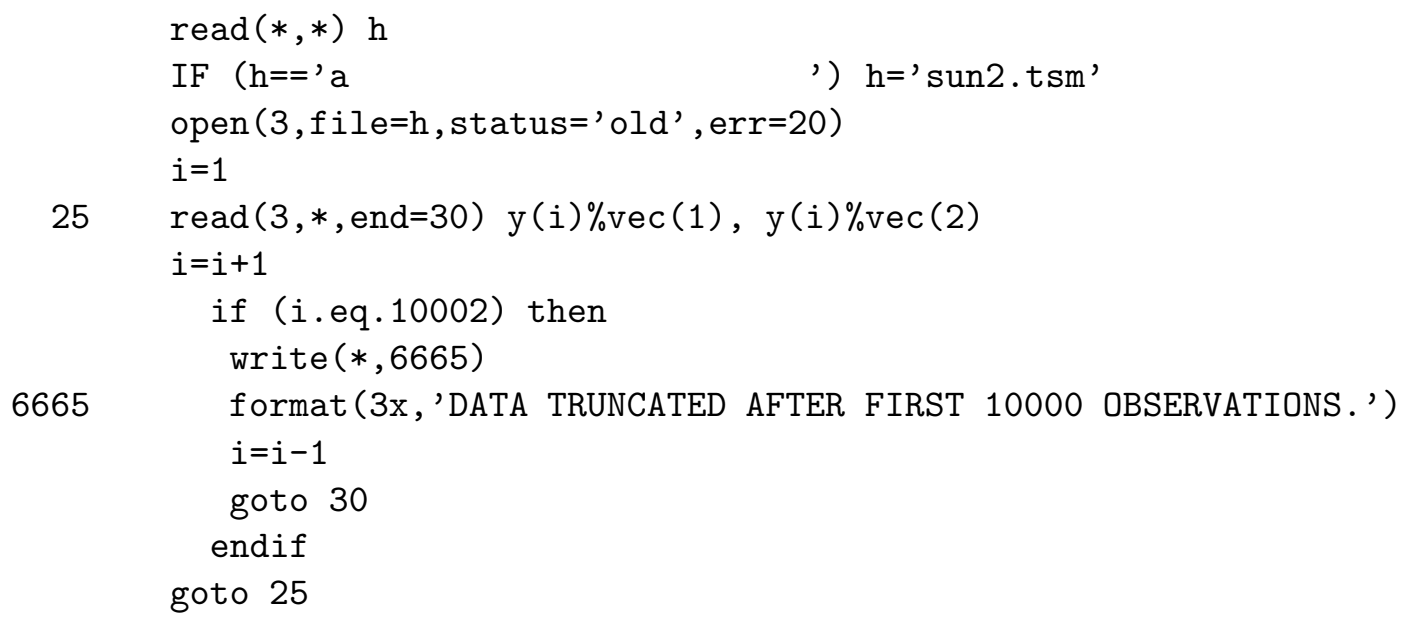




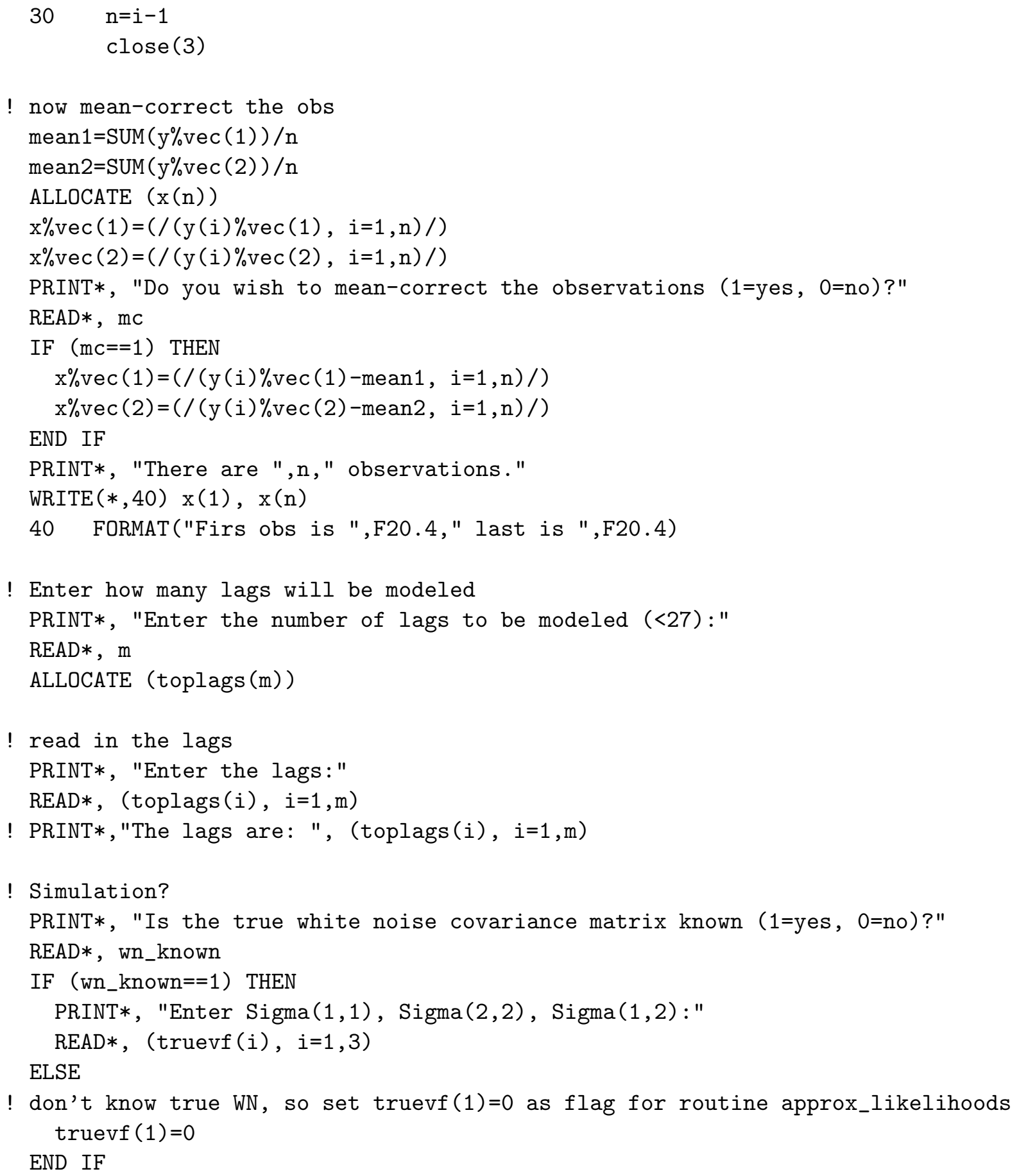




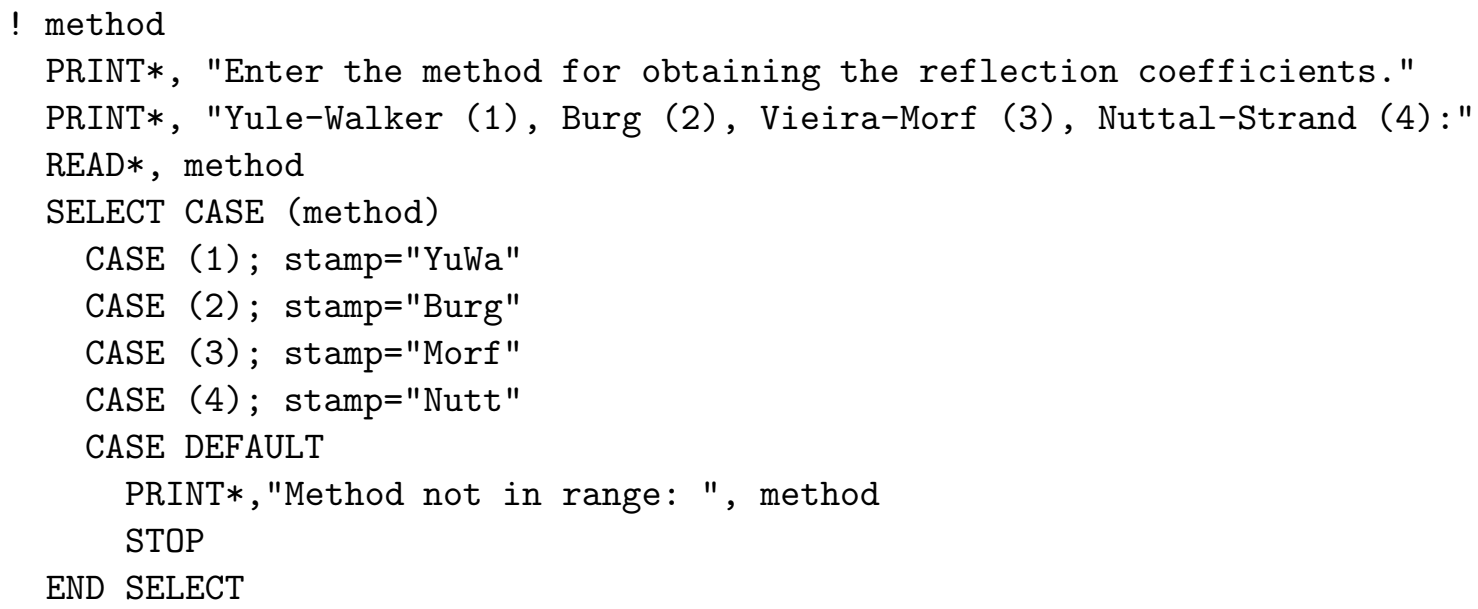

